\title{
Three-Dimensional Imaging With Multiple Degrees of Freedom Using Data Fusion
}

\section{This paper demonstrates that 3-D imaging using fusion provides multiple advantages including enhancement in visualization and reconstruction under different acquisition conditions.}

\author{
By Pedro latorre-Carmona, Filiberto Pla, Adrian Stern, Member Ieee, \\ Inkyu Moon, Member IEEE, And Bahram Javidi, Fellow IEEE
}

ABSTRACT | This paper presents an overview of research work and some novel strategies and results on using data fusion in 3-D imaging when using multiple information sources. We examine a variety of approaches and applications such as 3-D imaging integrated with polarimetric and multispectral imaging, low levels of photon flux for photon-counting 3-D imaging, and image fusion in both multiwavelength 3-D digital holography and 3-D integral imaging. Results demonstrate the benefits data fusion provides for different purposes, including visualization enhancement under different conditions, and 3-D reconstruction quality improvement.

KEYWORDS | Fusion; holography; integral imaging; multiresolution wavelet decomposition; multispectral imaging; photon counting; polarimetric imaging; 3-D imaging

\section{INTRODUCTION}

The definition given by the Joint Directors of Laboratories (JDL) about data fusion involves the "combination of data and information from several sources" [1]. This "require-

Manuscript received November 12, 2014; revised March 29, 2015; accepted June 25,2015 . The work of B. Javidi was supported by the National Science Foundation (NSF) under Grant IIS-1422179. The work of F. Pla was supported by the "Interactive visualiation 3D: Geometric models, graphics, learning and content generation" Project (PROMETEO-II/2014/062) and the "Técnicas de aprendizaje adaptativo y

caracterización en imágenes digitales para el reconocimiento automático de células sanguíneas" Project (P11B2014-09).

P. Latorre-Carmona and F. Pla are with the Institute of New Imaging Technologies, Universidad Jaume I, Castellón de la Plana 12071, Spain (e-mail: latorre@uji.es).

A. Stern is with the Department of Electro-Optics Engineering, Ben-Gurion University of the Negev, Beer-Sheva 84105, Israel.

I. Moon is with the School of Computer Engineering, Chosun University, Gwangju 501-759, Korea.

B. Javidi is with the Electrical and Computer Engineering Department, University of Connecticut, Storrs, CT 06269 uSA (e-mail: Bahram.Javidi@uconn.edu).

Digital Object Identifier: 10.1109/JPROC.2015.2451734 ment" was generalized in [2] when considering that the data could be provided either by a single source (sensor) or by multiple sources. In the particular case of image fusion methodologies, an intense research effort is being made in fields like remote sensing [3]-[7], surveillance [8]-[11], and medicine [12]-[15], where the current tendency is in the use of a combination of sensors to optimize information acquisition and extraction. In the case of remote sensing, the use of multiple sensors may help in rural and urban planning [3], species classification [4], [5], and prevention of disasters [6], [7].

On the other hand, surveillance systems may need to have all-weather and all-time acquisition capabilities. This capability might be given by fusing the information given by red-green-blue (RGB) and thermal sensors [8]-[10]. In the case of medicine there is a clear evidence that acquisition methodologies (magnetic resonance imaging, computed tomography, positron emission tomography, etc.) are complimentary and may be of great help in the prevention and detection of illnesses [11]-[15].

Other fields where image fusion from various data sources (sometimes referred as multimodal image fusion) is an active area of research include video processing [16], [17], biometric identification/classification [18]-[21], image fusion for visualization enhancement [22], industrial inspection [23], among others [24]-[27]. Image fusion methods might be also aimed at solving only one particular type of fusion problem, but current tendency is toward the creation of methodologies that may be able to solve image fusion problems in more than one area (fusion of medical images, or fusion of RGB and thermal images, using the same mathematical framework) [28]-[32].

Image fusion techniques can be classified taking into account the stage at which they are applied [33]. The most 
common strategy is to use the fusion strategy at feature level. However, they can also be designed to be used at the so-called decision level [34]. Fusion at the feature level means that the different features obtained are "combined" and later given to the stage where a final decision is taken. In the decision level case, different methods are simultaneously applied that provide different decisions on a local level, and the local decisions are combined using a decision function that creates the final decision.

In this paper, we present an overview of the work made by the authors on using fusion of different information sources in the particular framework of 3-D imaging. In our case, we consider fusion in the sense of using different degrees of freedom for light in order to improve information extraction. Besides, we can consider that the approaches explained here fall into the feature level fusion strategy [33].

To the best of our knowledge, a survey paper of different information sources (polarimetric, multispectral, different number of photons) and its combination in the framework of 3-D imaging has not been made to date. A general analysis of the state-of-the-art in multimodal image fusion is out of the scope of this paper, and the reader is referred to [33] about multimedia (including image and video) data fusion, as well as the different criteria that can be used to classify them, and [35] for multisensory data fusion. In particular, in this paper, we discuss multiperspective fusion under very low illumination conditions, 3-D imaging fusion with multispectral imaging, and multiwavelengths data fusion for digital holography and 3-D integral imaging. The paper is organized as follows. Section II presents an overview of integral imaging as an autostereoscopic technique that is currently being intensively researched. Section III presents multiperspective fusion results for very low illumination conditions. Section IV presents results on 3-D visualization at low light levels using multispectral photoncounting integral imaging. Section $\mathrm{V}$ presents new results on the fusion of 3-D information obtained from multiwavelengths integral images for robust depth extraction compared with a single-wavelength 3-D reconstructed image. Section VI presents an overview of multiwavelength data fusion for digital holography, and the fusion of polarimetric, multiwavelength and multispectral information for 3-D integral imaging.

In order to better understand the different concepts used in the paper, Table 1 presents a glossary of terms used throughout the paper, by order of appearance.

\section{INTEGRAL IMAGING OVERVIEW}

Three-dimensional optical image sensing and visualization technologies have been researched extensively for different applications in fields as diverse as TV broadcasting, entertainment, medical sciences, and robotics, to name a few [36]-[38]. As opposed to traditional 2-D imaging, 3-D sensing technologies can potentially capture the structural
Table 1 Glossary of Terms Used in This Paper

\begin{tabular}{|c|c|}
\hline Concept & Definition \\
\hline Auto-stereoscopy & $\begin{array}{l}\text { Group of techniques that "generate" 3-D } \\
\text { images for visualization purposes } \\
\text { without the use of special glasses }\end{array}$ \\
\hline Parallax barriers & $\begin{array}{l}\text { Device formed by a layer with vertical } \\
\text { slits that allows each eye to see a } \\
\text { different set of pixels and creating a } \\
\text { sense of depth. }\end{array}$ \\
\hline $\begin{array}{l}\text { Passive optical } \\
\text { device }\end{array}$ & $\begin{array}{l}\text { Optical device that does not use an } \\
\text { illumination system for image } \\
\text { acquisition. }\end{array}$ \\
\hline Stereo pair & $\begin{array}{l}\text { System that allows to present slightly } \\
\text { different images to each eye to create the } \\
\text { sense of depth. }\end{array}$ \\
\hline Elemental image & $\begin{array}{l}\text { Image generated by each one of the } \\
\text { lenslets. In the case of a moving sensor } \\
\text { in a Synthetic Aperture Integral Imaging } \\
\text { (SAII) mode, it corresponds to each one } \\
\text { of the images acquired by the sensor in } \\
\text { each of its positions. }\end{array}$ \\
\hline Lenslet array & $\begin{array}{l}\text { Array of small lenses (called lenslets) } \\
\text { located in a grid which allow to obtain } \\
\text { information from a scene from slightly } \\
\text { different view-points. }\end{array}$ \\
\hline $\begin{array}{l}\text { Points Spread } \\
\text { Function (PSF) }\end{array}$ & $\begin{array}{l}\text { Function that describes the response of } \\
\text { an optical system to a light point source } \\
\text { or object. }\end{array}$ \\
\hline $\begin{array}{l}\text { Depth of Focus } \\
\text { (DoF) }\end{array}$ & $\begin{array}{l}\text { Distance the image plane can be } \\
\text { displaced (plane where the image is } \\
\text { seen) while being this image in } \\
\text { acceptably sharp focus. }\end{array}$ \\
\hline Aberration & $\begin{array}{l}\text { Deviation from "normal operating } \\
\text { conditions" in an optical system. }\end{array}$ \\
\hline Field of View (FoV) & $\begin{array}{l}\text { Spatial/angular extent of an observable } \\
\text { scene that is seen by a sensor. }\end{array}$ \\
\hline Pickup aperture & $\begin{array}{l}\text { Opening of an optical system that } \\
\text { determines the light that arrives at the } \\
\text { sensor. }\end{array}$ \\
\hline Pinhole & $\begin{array}{l}\text { In optics, it refers to a small aperture the } \\
\text { light can go through. }\end{array}$ \\
\hline Bayer filter array & $\begin{array}{l}\text { It is a specific colour filter array to } \\
\text { arrange Red, Green and Blue Sensors on } \\
\text { a square grid. }\end{array}$ \\
\hline Holography & $\begin{array}{l}\text { Recording and display technique that } \\
\text { uses coherent light and generates images } \\
\text { that change its appearance with the } \\
\text { viewing angle. }\end{array}$ \\
\hline Optical path & $\begin{array}{l}\text { Path that the light follows when } \\
\text { propagates in a medium. }\end{array}$ \\
\hline $\begin{array}{l}\text { Fresnel } \\
\text { transformation } \\
\text { method }\end{array}$ & $\begin{array}{l}\text { Method to generate a discrete version of } \\
\text { the Fresnel double integral propagation } \\
\text { operator, used to assess its propagation } \\
\text { in any media. }\end{array}$ \\
\hline $\begin{array}{l}\text { Liquid Crystal } \\
\text { Spatial Light } \\
\text { Modulator } \\
\text { (LCSLM) }\end{array}$ & $\begin{array}{l}\text { A Spatial Light Modulator (SLM) is a } \\
\text { system that allows the generation of a } \\
\text { spatially varying modulation on a light } \\
\text { beam when the light beam passes } \\
\text { through it. It is called LC-SLM when the } \\
\text { element that creates the modulation is a } \\
\text { Liquid Crystal Device (LCD). }\end{array}$ \\
\hline
\end{tabular}

information of the scene. In many instances, the capabilities of 3-D imaging and display systems have revolutionized the progress of these disciplines, enabling new detection/display abilities that would not have been otherwise possible. There are different methods to create and 
visualize 3-D images. Some of them make use of special glasses, which may create adverse effects like sickness, etc. Other methods use parallax barriers in order to avoid using glasses, adapting their parameters to the viewer position [39]. Content-adaptive parallax barriers for which display elements are optimized for the multiview content are also being developed [40]. Alternative methods use multiple cameras located at several positions.

As one of the promising methods in the area of 3-D sensing and display, integral imaging is an autostereoscopic 3-D imaging method that offers a passive and relatively inexpensive way to capture 3-D information and to optically or computationally visualize it [41]-[46]. The integral imaging technique belongs to the broader class of multiview imaging techniques. Recently, it has been shown that the spatial 3-D resolution obtained with the integral imaging method is better than the resolution obtained when using a stereo pair [45]. Integral imaging provides 3-D information through a collection of 2-D projections of realworld objects. There are two separate procedures for acquisition and visualization of 3-D objects which are referred to as: 1) pickup and 2) reconstruction stages, respectively. In the pickup stage (see Fig. 1), multiple 2-D images (hereafter called elemental images) are captured through an array of small lenses (lenslet array). Each lenslet carries out a unique projective transformation that maps the 3-D object space onto a 2-D elemental image and is a function of the lenslet position and the focal length. Since each projective transform uniquely defines a perspective view, both direction and intensity of rays emanating from the 3-D object can be recorded on the image sensor. As a result, an array of inverted real images is

Lens array

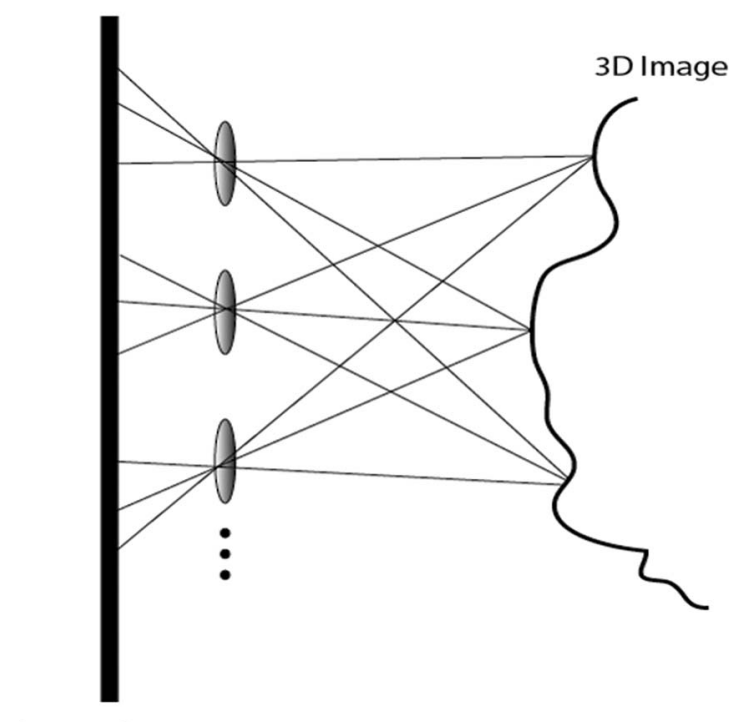

Image Sensor

Fig. 1. Integral imaging pickup procedure.

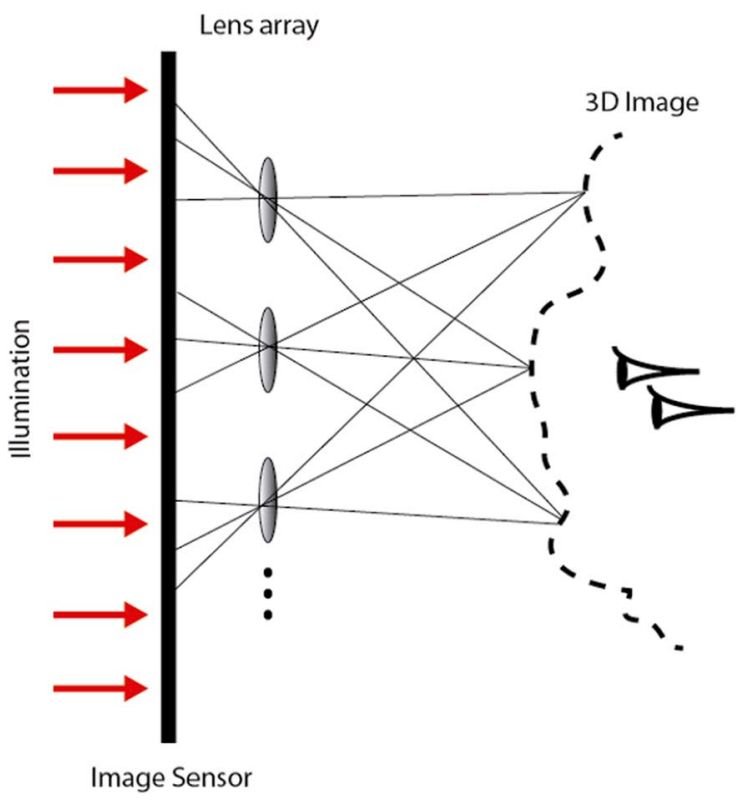

Fig. 2. Integral imaging display procedure.

formed on the image sensor. For optical reconstruction of the scene, a 2-D liquid-crystal display (LCD) may project the recorded integral image onto the focal plane of the display lenslet array with homogeneous (matched) pixel arrangement as that of pickup lenslet array. Each elemental image is then optically taken by its corresponding lenslet back into 3-D space. The overlap of all elemental images creates light distributions similar to the original object of interest. As a result, an observer can see a real 3-D image, with inverted depth (see Fig. 2).

In lenslet-based integral imaging systems, the resolution of each elemental image is constrained by three parameters: 1) the lenslet point spread function (PSF); 2) the lenslet depth of focus (DoF); and 3) the pixel size [47]-[49]. Aberrations and diffraction effects must also be considered because of the relatively small size of each lenslet.

As an alternative methodology to that based on lensletbased systems, integral imaging can be performed either using an array of high-resolution imaging sensors, or in a synthetic aperture mode, hereafter called synthetic aperture integral imaging mode (SAII; see Fig. 3). When a single sensor translates on a 2-D plane to capture multiple 2-D images, this enables one to obtain larger field-of-view (FOV) 2-D images. In addition, SAII potentially creates larger pickup apertures than the lenslet-array-based approach. Pickup aperture is useful for long-distance objects. Fig. 3 illustrates the pickup stage by using a sensor array.

Three-dimensional reconstruction of images can be achieved by computationally simulating the optical backprojection of the elemental images. In particular, a 


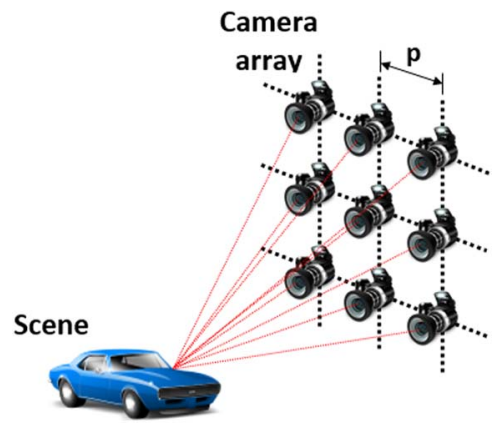

Fig. 3. Synthetic aperture integral imaging acquisition.

computer-synthesized virtual pinhole array is used to inversely map the elemental images into the object space, as illustrated in Fig. 4. Superposition of properly shifted elemental images provides the 3-D reconstructed images [50]

$$
I(x, y, z)=\frac{1}{O(x, y)} \sum_{k=0}^{K-1} \sum_{l=0}^{L-1} E_{k l}\left(x-k \frac{N_{x} p}{c_{x} M}, y-l \frac{N_{y} p}{c_{y} M}\right)
$$

where $I(x, y, z)$ represents the intensity of the reconstructed 3-D image at depth $z, x$ and $y$ are the indexes of the pixel, $E_{k l}$ represents the intensity of the $k$ th row and $l$ th column elemental image, $N_{x} \times N_{y}$ is the total number of pixels for each elemental image, $M=z / f$ is the magnification factor, $c_{x} \times c_{y}$ is the physical size of the camera sensor, $p$ is the camera pitch, and $O(x, y)$ is the overlapping number matrix.

The use of integral imaging techniques may be useful to recognize and classify objects under adverse conditions (in noisy or photon starved conditions), and under occlusions [51], [52], and recent results show the potential

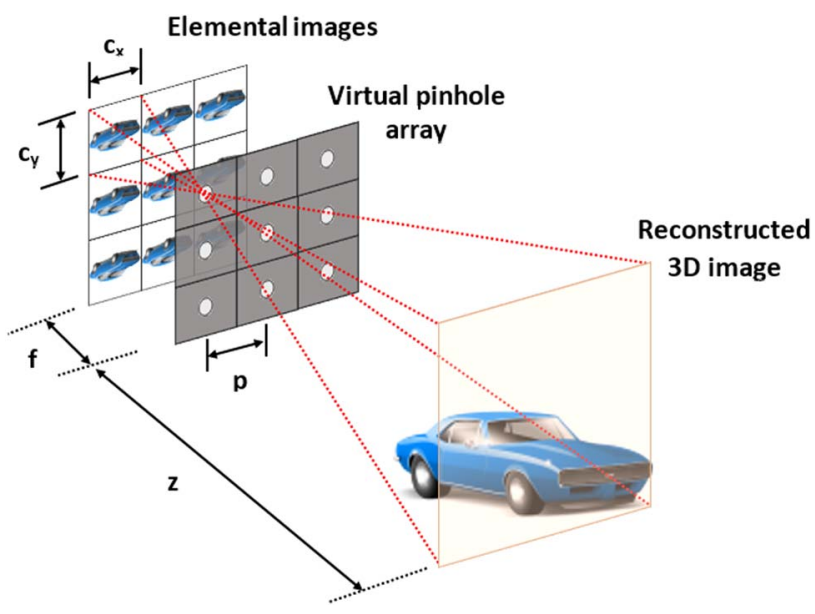

Fig. 4. Computational reconstruction method in integral imaging. of this technique in new application and research avenues [53]-[56].

\section{MULTIPERSPECTIVES FUSION TO IMAGE 3-D SCENES UNDER EXTREMELY LOW ILLUMINATION CONDITIONS}

Capturing images from multiple perspectives, as done with the integral imaging technique described in Section II, may be viewed as a multichannel image acquisition process. Since the data captured through the multiple channels (i.e., the perspective images) is typically redundant, it is possible by proper digital process, to overcome signal-to-noise ratio (SNR) limitations that are considered prohibitive with single-channel imaging. Fusion of multichannel images to overcome SNR limitation in 2-D imaging was explored in many fields as in astronomy, medical imaging, and microscopy. Here we describe the possibility to use the elemental images captured with the integral imaging technique to reconstruct 3-D objects in extremely starved imaging conditions. Photon starving imaging conditions may occur in scenarios of low light illumination, as in night vision, or when imaging with short exposure time, as for instance in high frame rate video acquisition.

The ability to visualize 3-D data from photon starved integral images was demonstrated in [57]. By applying the penalized maximum likelihood expectation-maximization (PMLEM) algorithm, 3-D images were reconstructed from integral images captured with SNR significantly lower than 1. For the description of the PMLEM reconstruction process, we will first formulate the integral imaging acquisition process using an operator formalism

$$
\mathbf{E}=\mathbf{H f}+\mathbf{n}
$$

where the 3-D object data are represented by the (lexicographically ordered) vector $\mathbf{f}, \mathbf{E}$ is a lexicographic vector representing the set of elemental images forming the integral image, $\mathbf{H}$ is the forward operator and $\mathbf{n}$ is the acquisition noise. Technically, the operator $\mathbf{H}$ can be implemented as a matrix or as a function handle (procedure) that maps each pixel from the object plane to the image plane. In the geometrical optics limits, $\mathbf{H}$ can be evaluated by simply tracing the ray from the object to the image plane (Fig. 1). If necessary, diffraction and other nongeometrical optical distortions can be included as well. In regular imaging conditions the image flux from the object is much higher than the noise (i.e., $\|\mathbf{f}\|>\|\mathbf{n}\|$ ) and a 3-D reconstruction can be obtained simply by a backprojection process. In an operator formalism this process is described as the application of the adjoint operator $\mathbf{H}^{\mathrm{T}}$ on the captured elemental images $\mathbf{E}, \mathbf{I}=\mathbf{H}^{\mathrm{T}} \cdot \mathbf{E}$. Technically, the backprojection process can be evaluated via (1). For photon starved conditions, an iterative reconstruction algorithm 
implementing a PMLEM estimator is much more efficient. The PMLEM iteration step is given by

$$
\hat{\mathbf{f}}^{k+1}=\frac{\hat{\mathbf{f}}^{(k)}}{\mathbf{s}+\beta \cdot \mathbf{P} \hat{\mathbf{f}}^{(k)}} \mathbf{H}^{\mathrm{T}} \frac{\mathbf{E}}{\mathbf{H} \cdot \hat{\mathbf{f}}^{(\mathrm{k})}}
$$

where $\hat{\mathbf{f}}^{(\mathrm{k})}$ is the reconstructed image vector at the $k$ th iteration, $\mathbf{s}$ is referred to as the "sensitivity vector" accounting for the number of elemental images contributing to reconstruction of each object point. $\mathbf{P}$ is the penalty operator and $\beta$ is a constant controlling the amount of regularization. The division in (3) should be taken as Hadamard (component-wise) division. The penalty operator $\mathbf{P}$ works as a regularizer to improve the robustness in case of large noise values. Various penalty operators can be defined, each reflecting a specific assumption on the local behavior of the object. In [58], penalties reflecting quadratic, median root, and total variation (TV) priors were investigated for photon-counting integral imaging. Among these priors the TV penalty was found to give the best results. The ability to overcome the noise limitation by fusing the elemental images captured with the integral imaging technique is demonstrated in Fig. 5. Fig. 5(a) shows an array of $7 \times 7$ elemental images captured with regular illumination conditions. Each elemental image captures from slightly different direction the 3-D scene which comprised two toys: a truck and an airplane located at distances of 50 and $70 \mathrm{~cm}$ from the camera, respectively. Fig. 5(b) and (c) shows the reconstruction of the 3-D scene by focusing at two depth planes of the two toys. The reconstruction is implemented by backprojection [see (1)]. Fig. 5(d) shows the array of elemental images taken with the same geometrical setup as that used for capturing Fig. 5(a), but with a shorter exposure time so that the SNR (defined as $\|E\| /\|n\|)$ is approximately 0.16 . With such a low SNR the signal is buried much below the noise level, therefore the objects are absolutely indistinguishable. However, by fusing the elemental images using the PMLEM algorithm with a TV prior the two objects, at the two different depth planes, can be readily reconstructed, as demonstrated in Fig. 5(e) and (f).

\section{THREE-DIMENSIONAL VISUALIZATION AT LOW LIGHT LEVEL USING MULTISPECTRAL PHOTON-COUNTING INTEGRAL IMAGING}

Photon-counting imaging technique which is suitable to photon-limited situations such as night vision has been successfully applied in 3-D object imaging and recognition [59]-[62]. On the other hand, 3-D objects can be computationally reconstructed and recognized in a photoncounting integral imaging (PCII) system with grayscale elemental images under low-light levels [59]. However,
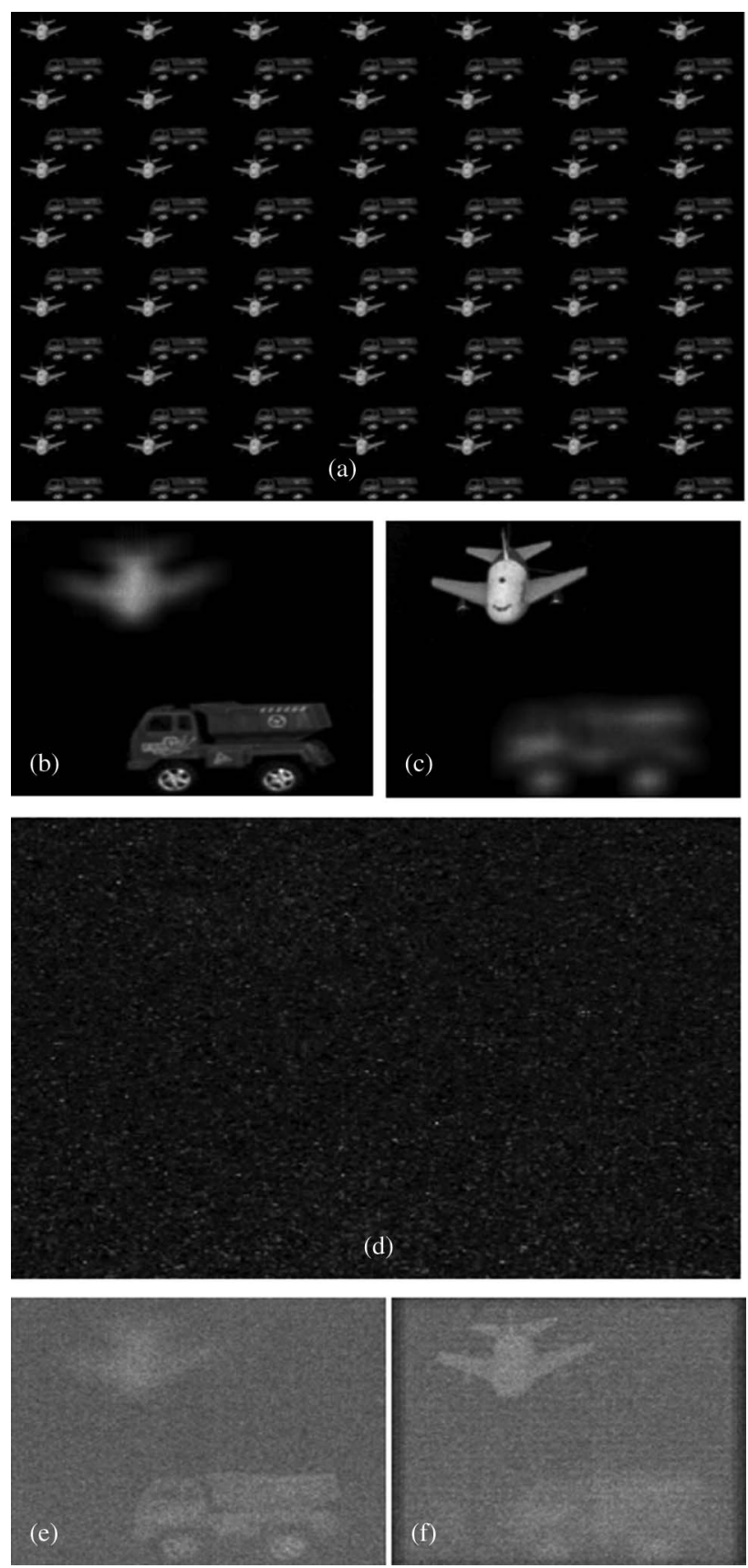

Fig. 5. (a) $7 \times 7$ elemental images, and reconstruction of object planes at (b) a distance of $50 \mathrm{~cm}$ and (c) a distance of $70 \mathrm{~cm}$. (d) $7 \times 7$ elemental images captured in photon starved conditions (SNR $=0.16$, average flux of 3.8 photons/pixels) and reconstructed object planes at distance $50 \mathrm{~cm}(e)$ and $70 \mathrm{~cm}$. (f) All the results are from [57].

the elemental images in this PCII system are captured with monochrome sensors and the object boundaries might be easily degraded when they are imaged under low light levels. Similarly, the reconstructed object in a PCII system may also suffer from degraded shape cues. As a consequence, object recognition based on these degraded images is a challenging problem. Nevertheless, color image 


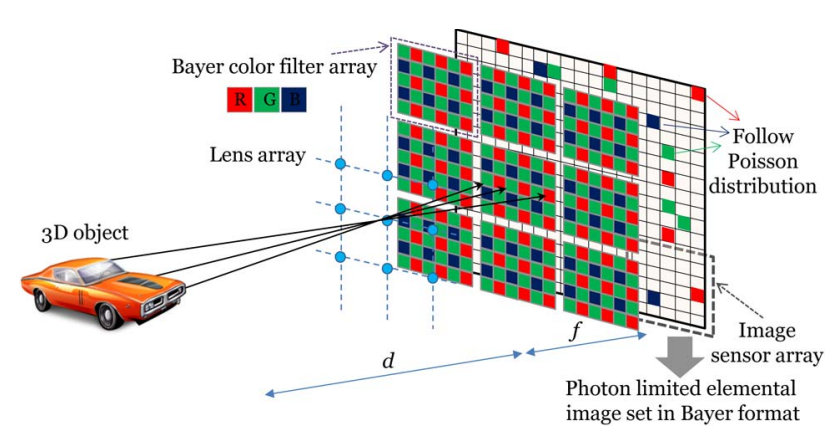

Fig. 6. Schematic setup of multispectral PCII system shown in [63].

information can provide informative patterns to help us visualize 3-D objects. In addition, color information may improve recognition performance when other features are not significant such as degraded shape cues in a photoncounting integral imaging system. Therefore, multispectral photon-counting integral imaging system may ameliorate the deficiency that exists in conventional PCII with grayscale elemental images since these images may lose an important amount of information of detail of the object. In [63], we have demonstrated that a 3-D scene can be visualized at a low light levels with our proposed multispectral PCII technique.

The diagram of multispectral PCII system is given in Fig. 6. A Bayer color filter array which is a widely adopted color image sensor is used to capture the color information of 3-D objects. However, a demosaicing or interpolation algorithm has to be introduced to estimate the missing two colors in a Bayer image in order to get a full RGB color image. Our numerical simulation results showed that the gradient corrected linear interpolation method proposed by Malvar et al. [64] may achieve better results for demosaicing Bayer image into full RGB color image when compared with some of the state-of-the-art demosaicing schemes [63]. The Malvar et al.'s interpolation method uses the interpolated current pixel value and the gradient information in the Bayer image to estimate the two missing values at the current pixel location.

In Fig. 6, multiple Bayer color filter arrays are used to capture Bayer elemental images in the integral imaging sys tem. However, when all of the Bayer elemental images are generated under low light level conditions, the photoncounted Bayer elemental images would be recorded in Bayer color filter arrays in this multispectral PCII system. This process can be simulated by controlling the expected number of photons in a multispectral scene. It is assumed that each pixel separately follows a Poisson distribution for each channel (R, G, and B) of the Bayer image. Assume that $\tilde{B}_{w}(x, y)$ is the normalized irradiance of each color channel on the Bayer image and $n_{p}$, the expected number of photons for each elemental image, then the Poisson parameter $\lambda_{w}$ for each pixel on the three color channels can be written as $\tilde{B}_{w}(x, y) \times n_{p}$. Thus, the Poisson random number of counts for arbitrary pixel positions on each elemental image is modeled as follows [63]:

$$
C_{w}(x, y) \approx \operatorname{Poisson}\left(\lambda_{w}=\tilde{B}_{w}(x, y) \times n_{p}\right)
$$

where the subscript $w$ represents the three RGB color channels. On the other hand, the 3-D point is computationally reconstructed with the photon-counted Bayer elemental images by using parametric maximum likelihood estimator (MLE) [58]. That is, the 3-D point can be achieved with MLE by using a series of 2-D points on the photon-counted Bayer elemental images. This process can be modeled as follows [63]:

$$
\begin{aligned}
P S_{w}(v) & =\operatorname{MLE}\left(\lambda_{w}(v)\right) \\
& =\underset{\lambda_{w}(v)}{\arg \max }\left(\log \prod_{k=1}^{N} \exp \left(-\lambda_{w}(v)\right)\left(\lambda_{w}(v)\right)^{C_{k, w}(v)}\right) \\
& =\frac{1}{N} \sum_{k=1}^{N} C_{k, w}(v)
\end{aligned}
$$

where $C_{k, w}(v)$ is the pixel point value of the $k_{t h}$ photoncounted elemental image. $P S_{w}(v)$ represents the 3-D scene, $v$ defines one voxel position, $\lambda_{w}(v)$ denotes the mean parameter of the $w$ (Red, Green, or Blue) spectral channel, and $N$ denotes the total number of elemental images. Finally, the reconstructed Bayer slice image is demosaiced with the gradient corrected linear interpolation technique.

Experiments were conducted to show the feasibility of the multispectral PCII system for 3-D visualization at low light level conditions. Two different objects (three colored balls and a toy bird) were used as the 3-D scene. With these Bayer elemental images, the two 3-D objects with Bayer format can be computationally reconstructed at two different distances. Then, the true color RGB reconstructed images can be achieved by applying Malvar's demosaicing algorithm to these reconstructed Bayer images. Fig. 7 shows the reconstructed Bayer images and their corresponding true color RGB images after Malvar's demosaicing method is applied.

In the case of low light levels, the photon-counted Bayer elemental images can be simulated based on Bayer elemental images using (4) and controlling the expected number of photons. With these photon-counted Bayer elemental images simulated under different number of photons, we show in Fig. 8 that the multispectral 3-D objects can be correctly visualized. The images in Fig. 8 were demosaiced from the reconstructed Bayer images that are derived with (5) by using these photon-counted Bayer elemental images at two different distances where one is with the color balls in focus, while the other one is with toy bird in focus. 

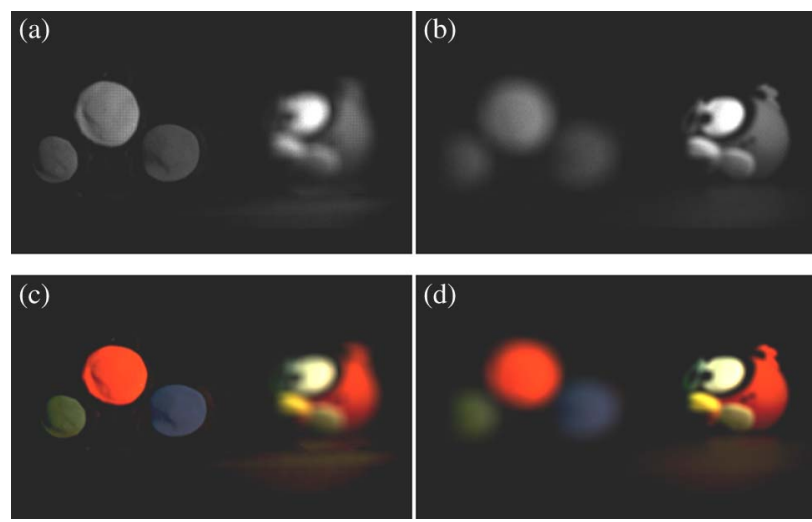

Fig. 7. Reconstructed images at two different depths.

(a) and (b) Reconstructed Bayer images with different object focused. (c) and (d) True color RGB images of (a) and (b) with Malvar's interpolation method applied [63].

Fig. 8 also shows that this multispectral PCII method can be used for multispectral visualization of 3-D objects when the number of photons is limited. In addition, the following PSNR metric was applied to measure the quality of multispectral 3-D reconstructed images under low light levels:

$$
\text { PSNR }=10 \log _{10}\left(\frac{I_{\max }^{2}}{M S E}\right)
$$
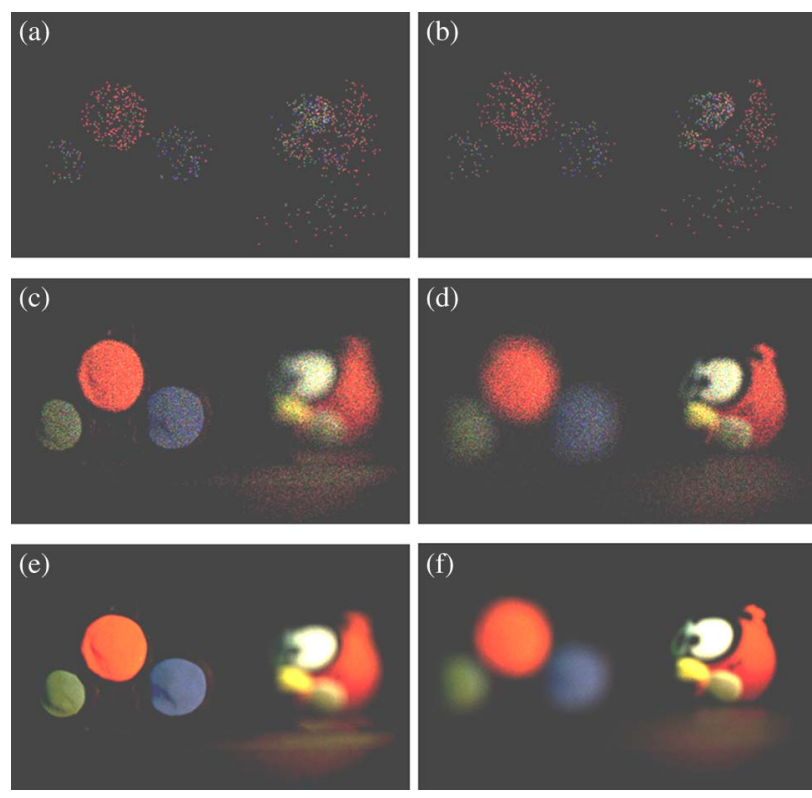

Fig. 8. Multispectral 3-D visualization under low light levels. (a), (c), and (e) are reconstructed RGB images with tricolored balls focused under $n_{p}=10,10^{3}$, and $10^{5}$ respectively. (b), (d), and (f) are reconstructed RGB images with toy bird focused under $n_{p}=10,10^{3}$, and $10^{5}$, respectively [63].
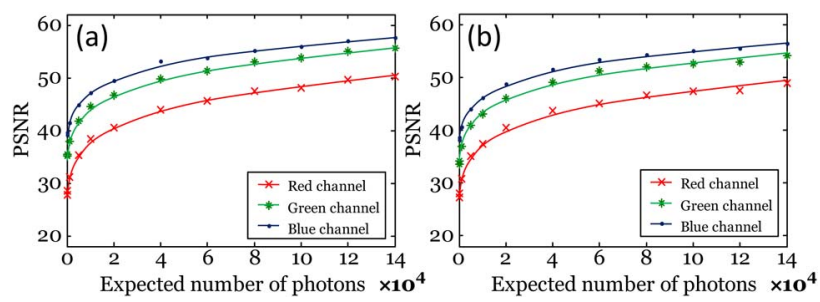

Fig. 9. PSNR [as defined in (6)] for green, red, and blue channels between intensities of multispectral II $\left(I_{\mathrm{MII}}\right)$ in Fig. 7 and multispectral photon-counting II $\left(I_{\mathrm{MPCI}}\right)$ in Fig. 8 versus the expected number of photons. (a) PSNR between Fig. 7 (c) and $I_{\mathrm{MPCII}}$ in Fig. 8 (focused ball images). (b) PSNR between Fig. $7(d)$ and $I_{\mathrm{MPCI}}$ in Fig. 8 (focused bird images) [63].

where $I_{\max }$ is the maximum possible pixel value of the multispectral integral imaging as ground truth or reference $\left(I_{\mathrm{MII}}\right)$ and MSE is mean squared error between multispectral photon-counting integral imaging (IMPCII) and $I_{\mathrm{MII}}$. It quantitatively verified that the multispectral 3-D scene can be reconstructed from photon-counted Bayer elemental images with MLE and gradient corrected linear interpolation algorithm at low light levels (see Fig. 9 and [63]).

\section{MULTISPECTRAL DATA FUSION FOR DEPTH ESTIMATION IMPROVEMENT IN 3-D INTEGRAL IMAGING}

Introduction of spectral information may help in object recognition and/or classification in 3-D imaging: 1) in underwater 3-D visualization [51], since water absorption is wavelength dependent; 2) in dermatology [65], in particular in skin cancer detection, because melanoma pigmented skin lesion is wavelength dependent and its structure is directly related to its evolution and degree of severity; 3) in remote sensing applications, in the case of sensors onboard airplanes or satellites which may be able to create 3-D models with the inclusion of multispectral information; 4) in remote sensing pattern recognition (i.e., identification of the 3-D structure and the spectral response of an object from the distance); and 5) in photon starved or "hard to visualize" conditions (at night, under "foggy" conditions, etc.) [60]. Multispectral 3-D reconstruction has already been applied in microscopy [66]. Other 3-D acquisition and visualization techniques have already incorporated multispectral information [67]-[69].

In this section, we will show novel results in the fusion of integral images extracted from different spectral channels, combining the depth information of each multispectral channel to make more accurate and robust depth estimation in scenes acquired with a multispectral sensor.

Three-dimensional image reconstruction in integral imaging has been traditionally performed in orthogonal 


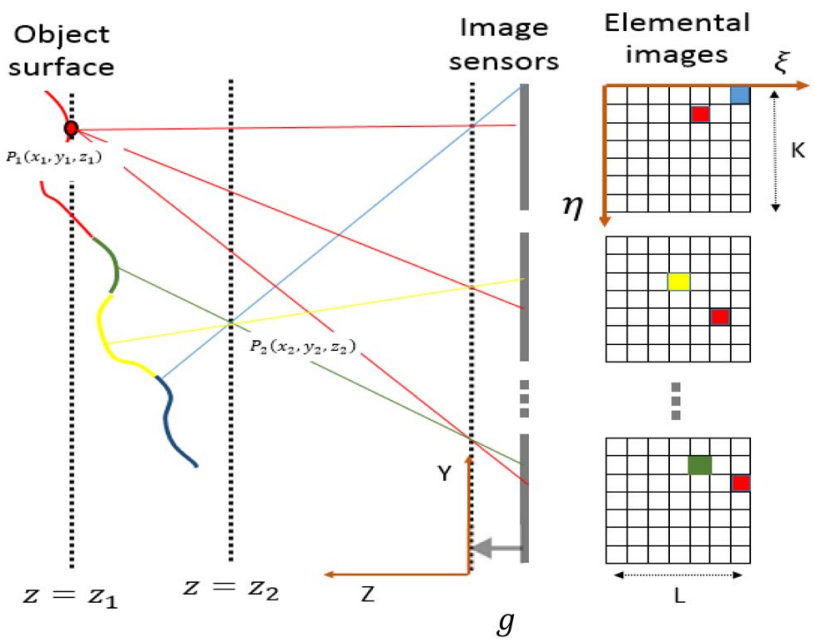

Fig. 10. Variance minimization principle (adapted from [71]).

planes to the optical axis. A certain spatial point on the reconstruction plane is set to the average of the collected samples of the corresponding 3-D space point. Based on the pinhole projection model, the relationship between the coordinate of 3-D space point $(x, y, z)$ and its projected image pixel coordinate $(\xi, \eta)$ on the ith column and jth row image sensor is given by [70]

$$
(\xi, \eta)=\left(\frac{K g\left(x+C_{x}^{i j}\right)}{s_{x} z}, \frac{\operatorname{Lg}\left(y+C_{y}^{i j}\right)}{s_{y} z}\right)
$$

where superscript ij means the ith column and the $j$ th row, $\left(C_{x}^{i j}, C_{y}^{i j}\right)$ is the center position of the ijth image sensor, $\left(S_{x}, S_{y}\right)$ is the image size, and $g$ represents the distance between the pinhole and the image sensor (see Fig. 10).

From this information, depth of each point in the scene can be estimated using the method explained in [71] and summarized here. Let us define the statistical mean and variance for a 3-D point $P(x, y, z)$ as follows:

$$
\begin{aligned}
E(x, y, z) & =\frac{\sum_{i=1}^{N} \sum_{j=1}^{M} I^{i j}(\xi, \eta)}{N M} \\
V(x, y, z) & =\frac{\sum_{i=1}^{N} \sum_{j=1}^{M}\left(I^{i j}(\xi, \eta)-E(x, y, z)\right)^{2}}{N M}
\end{aligned}
$$

where $I^{i j}(\xi, \eta)$ represents the intensity of pixel $(\xi, \eta)$ on the ijth elemental image, and $N$ and $M$ represent the number of elemental images in each $(x$ and $y)$ direction. $E(x, y, z)$ and $V(x, y, z)$ represent the intensity mean and variance of 3-D point, respectively. Therefore, if a point belongs to an object, $V(x, y, z)$ is expected to reach a local minimum
(Fig. 10). Therefore, the depth of each 3-D object point can be estimated by considering

$$
\hat{z}(x, y)=\arg \min V(x, y, z)
$$

with $z \in z=\left[z_{\min }, z_{\max }\right]$. This method, however, suffers from one main drawback. The variance does not "behave" correctly for parts of objects in the scene that present specular reflection or surface sharp discontinuities. The method proposed here to overcome this drawback is to take advantage of the focusing capability in synthetic aperture integral imaging and therefore also use a "focus measure" criterion. The "Tenengrad" focus measure method [72] consists of assessing the following function per pixel position:

$$
\phi(x, y)=\sum_{(i, j) \in \Omega(x, y)}\left(G_{x}(i, j)^{2}+G_{y}(i, j)^{2}\right)
$$

where $\Omega(x, y)$ represents the group of pixels in a neighborhood of pixel $(x, y)$, and $G_{x}=I \otimes S_{x}$ and $G_{y}=I \otimes S_{y}$ are the " $x$ " and " $y$ " image gradients obtained by convolving an image (I) with a Sobel filter in the " $x$ " and " $y$ " directions $\left\{S_{x}, S_{y}\right\}$.

In practice, depth estimation varies if the scene is acquired with spectral filters. When combining multispectral and integral imaging, a straightforward way to perform 3-D reconstruction is to apply the process to each multispectral band independently. However, we can take advantage of the 3-D information each band may acquire and combine them to improve the scene depth measurements obtained from each spectral band independently.

\section{A. Depth Estimation Using a Combination of Variance and Focus Measure}

A series of 121 elemental images were acquired using a Marlin F080B monochrome camera and a liquid crystal tunable filter (LCTF) applying the SAII technique, for a $11 \times 11$ grid [73]. Acquisition wavelengths were: $\{480,510,550$, $570,600,650,680\}$. Fig. 11(a) shows the experimental setup used for the acquisition. Fig. 11(b) shows the grayscale image corresponding to the elemental image in position $(6,5)$ of the $11 \times 11$ grid, for $\lambda=650 \mathrm{~nm}$. The proposed multispectral fusion strategy consists of the following steps.

1) Infer the depth corresponding to the minimum variance and the depth of the maximum Tenengrad function measure for each pixel and wavelength band

$$
\begin{aligned}
& z_{1}^{*}\left(x, y, \lambda_{i}\right)=\arg \min _{z} V\left(x, y, z, \lambda_{i}\right) \\
& z_{2}^{*}\left(x, y, \lambda_{i}\right)=\arg \max _{z} \phi\left(x, y, z, \lambda_{i}\right) .
\end{aligned}
$$

2) Select for each pixel the depth corresponding to the minimum variance among bands, and the 


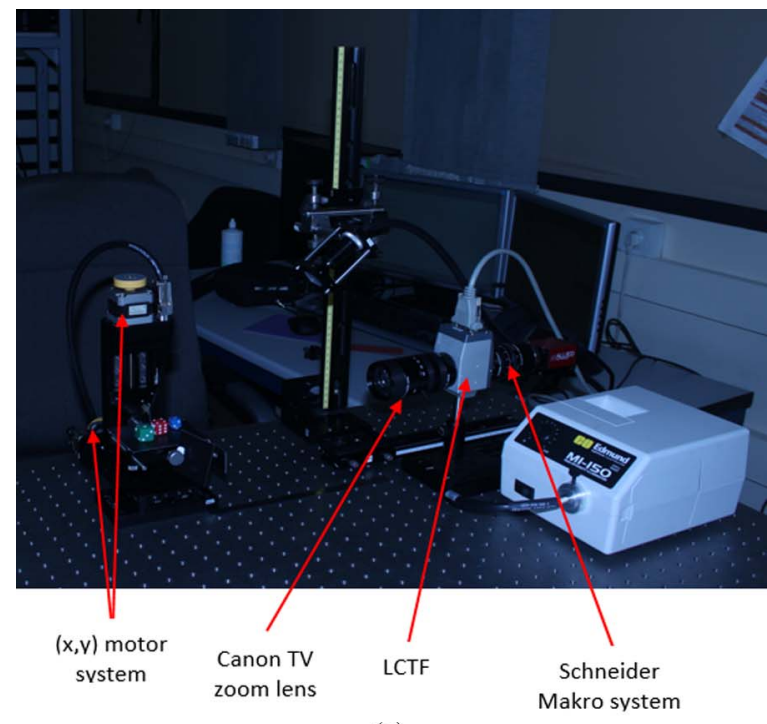

(a)

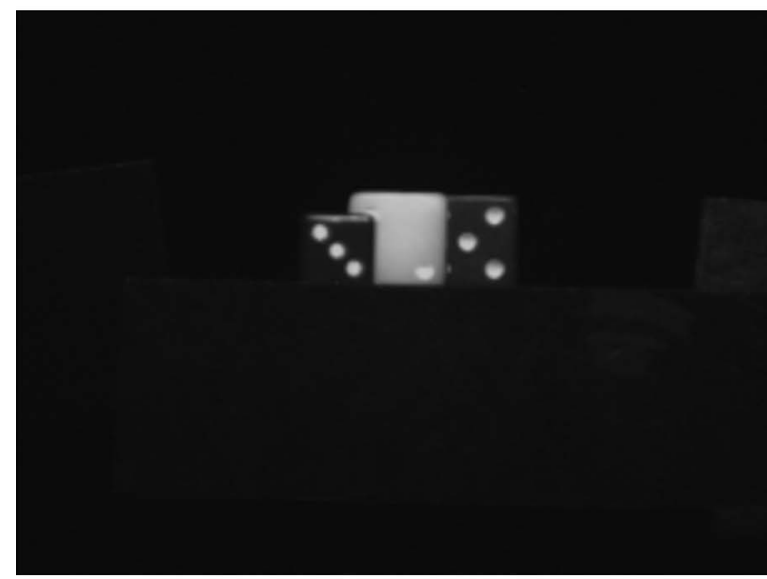

(b)

Fig. 11. (a) Experimental setup for the multispectral integral imaging system shown in [73]. Elemental image in position $(6,5)$ of the camera array for $\lambda=650 \mathrm{~nm}$.

depth corresponding to the maximum focus measure among bands as well

$$
\begin{aligned}
& z_{1}^{*^{\prime}}(x, y)=\arg \min _{\lambda_{i}}\left[\mathrm{~V}\left(x, y, z_{1}^{*}\left(x, y, \lambda_{i}\right), \lambda_{i}\right)\right] \\
& z_{2}^{*^{\prime}}(x, y)=\arg \max _{\lambda_{j}}\left[\phi\left(x, y, z_{2}^{*}\left(x, y, \lambda_{j}\right), \lambda_{j}\right)\right] .
\end{aligned}
$$

3) Infer the variance histogram corresponding to the minimum variance distribution among bands of step 2) and generate a variance threshold $(\theta)$ corresponding to $99.5 \%$ of the accumulated variance in this histogram

$$
\theta\left[H\left(V\left(z_{1}^{*^{\prime}}(x, y)\right)\right)\right]=\tilde{v}
$$

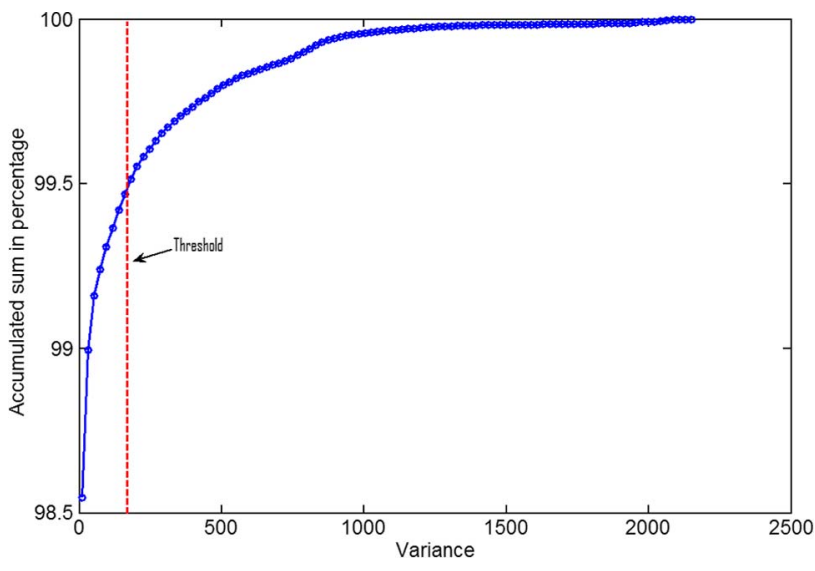

Fig. 12. Accumulated histogram for the variance for the depths obtained using (14). The line corresponding to the threshold used in (17) is plotted as well.

such that

$$
\frac{\sum_{b=1}^{\widetilde{V}} H(V)}{V_{T}}=0.995
$$

where $V_{T}=\sum_{D} H(V)$ is the total accumulated variance for the whole variance range.

4) If the variance of a pixel [in the histogram of step 3)] is higher than $\theta$, the distance associated to the pixel is the one given by the "focus measure" criterion in step 2). Otherwise, the minimum variance in 2) is associated to this pixel

$$
z_{F}(x, y)= \begin{cases}z_{2}^{*^{\prime}}, & \text { if } V\left(x, y, z_{1}^{*^{\prime}}\right) \geq \theta \\ z_{1}^{*^{\prime}}, & \text { otherwise }\end{cases}
$$

Fig. 12 shows the accumulated histogram distribution of $H\left(V\left(z_{1}^{z^{\prime}}(x, y)\right)\right)$. The threshold $\theta$ corresponding to the $99.5 \%$ of the accumulated variance is represented as a dotted red line. Table 2 shows the measured distance from each one of the dice to the Canon TV zoom lens. Table 3 shows the depth estimation results obtained using: a) one wavelength $(\lambda=650 \mathrm{~nm})$; and $\mathrm{b})$ the procedure explained in steps 1)-4).

We considered that the best depth reconstruction for a single wavelength was that at $\lambda=650 \mathrm{~nm}$, which is the

Table 2 Distance From Each Die to the Acquisition System

\begin{tabular}{|c|c|c|}
\hline & & \\
\hline Blue die $(\mathrm{mm})$ & Red die $(\mathrm{mm})$ & Green die $(\mathrm{mm})$ \\
\hline 220 & 240 & 262 \\
\hline
\end{tabular}


Table 3 Distance Estimation Results for the Best Wavelength Case and for the Different Fusion Methods

\begin{tabular}{|l|c|c|c|}
\hline & Blue die $(\mathrm{mm})$ & Red die $(\mathrm{mm})$ & Green die $(\mathrm{mm})$ \\
\hline$\lambda=650 \mathrm{~nm}$ & $237.8 \pm 18.7$ & $254.7 \pm 13.3$ & $265.1 \pm 20.8$ \\
\hline Fusion & $238.1 \pm 7.7$ & $249.6 \pm 10.6$ & $262.4 \pm 6.1$ \\
\hline
\end{tabular}

one with the lowest total RMSE error defined as

$$
\operatorname{RMSE}=\sqrt{\frac{1}{N} \cdot \sum_{i=1}^{N}\left(d_{i}-d_{G T, i}\right)^{2}} .
$$

Here $d_{i}$ refers to the distance between pixel " $i$ " and the acquisition system, as assessed by a method and $d_{G T, i}$ refers to the ground-truth distance measured by a rule.

Table 4 shows the total RMSE as well as RMSE error per die.

Fig. 13(a) shows a 3-D plot with the depth reconstruction results for $\lambda=650 \mathrm{~nm}$ obtained using the minimum variance criterion in (9). Fig. 13(b) shows the corresponding depth results after the application of the formulas given in (12)-(17). As we can see, the problems that appear for the depth reconstruction using the minimum variance principle are not so evident. Fig. 13(c) shows the application of steps 1 and 3 of this strategy, for $\lambda=650 \mathrm{~nm}$. In the three cases, the grayscale image of elemental image $(6,5)$ for $\lambda=650 \mathrm{~nm}$ has been used for visualization purposes.

\section{IMAGE FUSION IN}

\section{MULTIWAVELENGTH DIGITAL}

\section{HOLOGRAPHY AND INTEGRAL IMAGING}

This section shows the results obtained by the authors in the application of the multiresolution wavelet decomposition (MWD) technique for image fusion in two different fields: 1) multiwavelength digital holography (MWDH); and 2) multidimensional optical data fusion, showing its capability to improve visualization results in both cases.

\section{A. Multiwavelength Digital Holography}

Digital holography (DH) [74]-[91] has been applied in different fields such as microscopy [74]-[75], imaging and display technology [76]-[77], data storage [78], 3-D object recognition and 3-D optical encryption [79], [80], to cite a few. Current research efforts also focus on including color and multispectral information in DH [92]-[100]. The

Table 4 Total Root Mean Square Error $\left(\mathrm{RMSE}_{T}\right)$ and RMSE Error Per Die

\begin{tabular}{|l|c|c|c|c|}
\hline & $R M S E_{T}$ & $R M S E_{B}$ & $R M S E_{R}$ & $R M S E_{G}$ \\
\hline$\lambda=650 \mathrm{~nm}$ & 21.6 & 24.5 & 19.9 & 20.8 \\
\hline Fusion & 14.0 & 19.8 & 14.3 & 7.1 \\
\hline
\end{tabular}

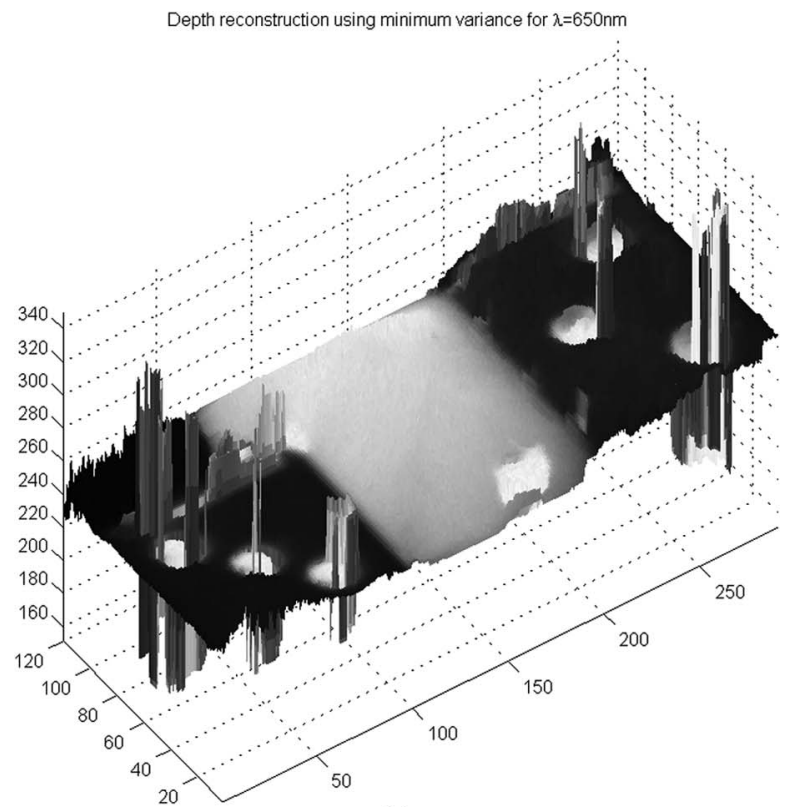

(a)

Depth reconstruction for the combination of the minimum variance and focus measure principles

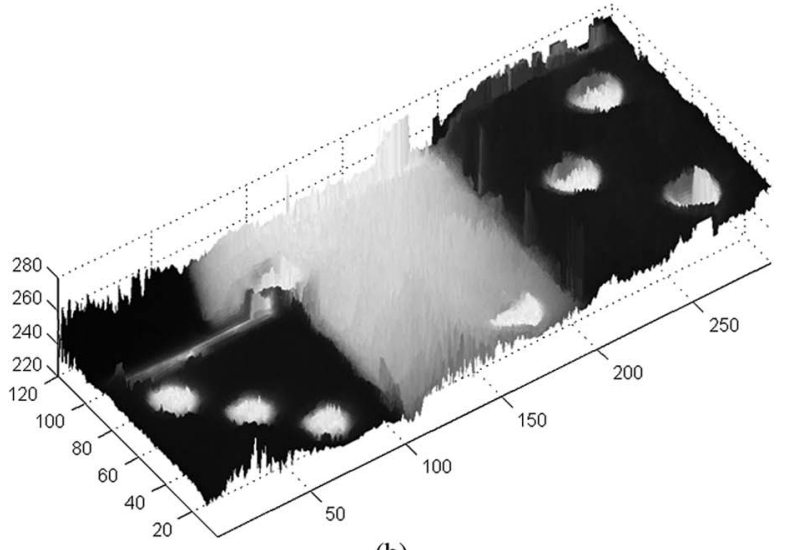

(b)

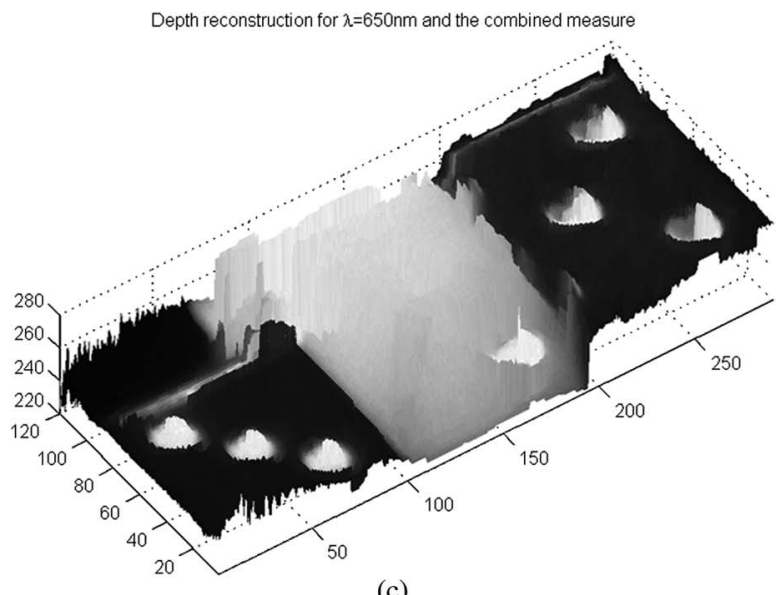

(c)

Fig. 13. (a) Depth reconstruction result using the minimum variance principle. (b) Depth reconstruction for the combination of the minimum variance and the focus measure strategy in steps 1-4 in Section v. (c) Depth reconstruction when using criteria in (b) for $\lambda=650 \mathrm{~nm}$ only. 
interested reviewer is referred to [101] for a comprehensive review for current fields of application.

In this section, a summary of the fusion results shown in [102] will be presented. Multiwavelength digital holography (MWDH) has been extensively researched during the last few years. It has shown superior object phase recovery capability in relation to other techniques [101], natural color holographic cameras have already been proposed [90], [91], and a great effort has been made to infer the spectral information of objects simultaneously to capture 3-D information using DH [91].

Image fusion methods aim at creating a composite image of the same scene acquired at different wavelengths. Gaussian pyramids and multiresolution wavelet decomposition (MWD) techniques have received attention during the last decade.

MWD may be combined with MWDH in order to generate better quality color 3-D images. Direct RGB creation of reconstructed images acquired at different wavelengths has contrast and resolution limitations, due to the speckle noise, which appears when using coherent light illumination. MWD techniques are based on the decomposition of the lowfrequency component of the previous resolution level and its assignment into the higher resolution level. Let us consider $P_{0}$ a grayscale image. The first level decomposition will be

$$
P_{0} \longrightarrow P_{a 1}, P_{v 1}, P_{h 1}, P_{d 1}
$$

$P_{a 1}$ is the so-called approximation term, and $P_{v 1}, P_{h 1}$, and $P_{d 1}$ are the vertical, horizontal, and diagonal details of the next (first in this case) level decomposition, respectively. The details and the approximation contain the high-frequency and low-frequency features of the image, respectively. The approximation of each level may be recursively decomposed into the next-level components as follows:

$$
P_{a(n-1)} \longrightarrow P_{a n}, P_{v n}, P_{h n}, P_{d n}, \quad \text { for } n=1,2, \ldots
$$

Once the multiresolution wavelet coefficients of each image acquired by each sensor are obtained, high- and lowfrequency components can be postprocessed using specific fusion rules, such as addition, or weighted averaging. Fig. 14 shows a two-level MWD fusion method for multiwavelength images. These images need to be registered. After registration, their multiresolution wavelet decomposition is obtained. Fusion rules are applied to their high- and low-frequency components afterwards. These transformed (fused) wavelet coefficients are inverse wavelet transformed into a final fused image.

The experimental setup used to record MWDH holograms is illustrated in Fig. 15. Two light sources consisting of two lasers emitting at $\lambda_{1}=632.8 \mathrm{~nm}$ and $\lambda_{2}=532.0 \mathrm{~nm}$ were used. The two lasers were allowed to propagate along the same path for either the reference or the object beams. The reflecting prism located in the path of the red laser beam allows matching the optical paths of the two interfering beams inside the optical coherence length of the laser. The object beam was at a distance of $850 \mathrm{~mm}$ in

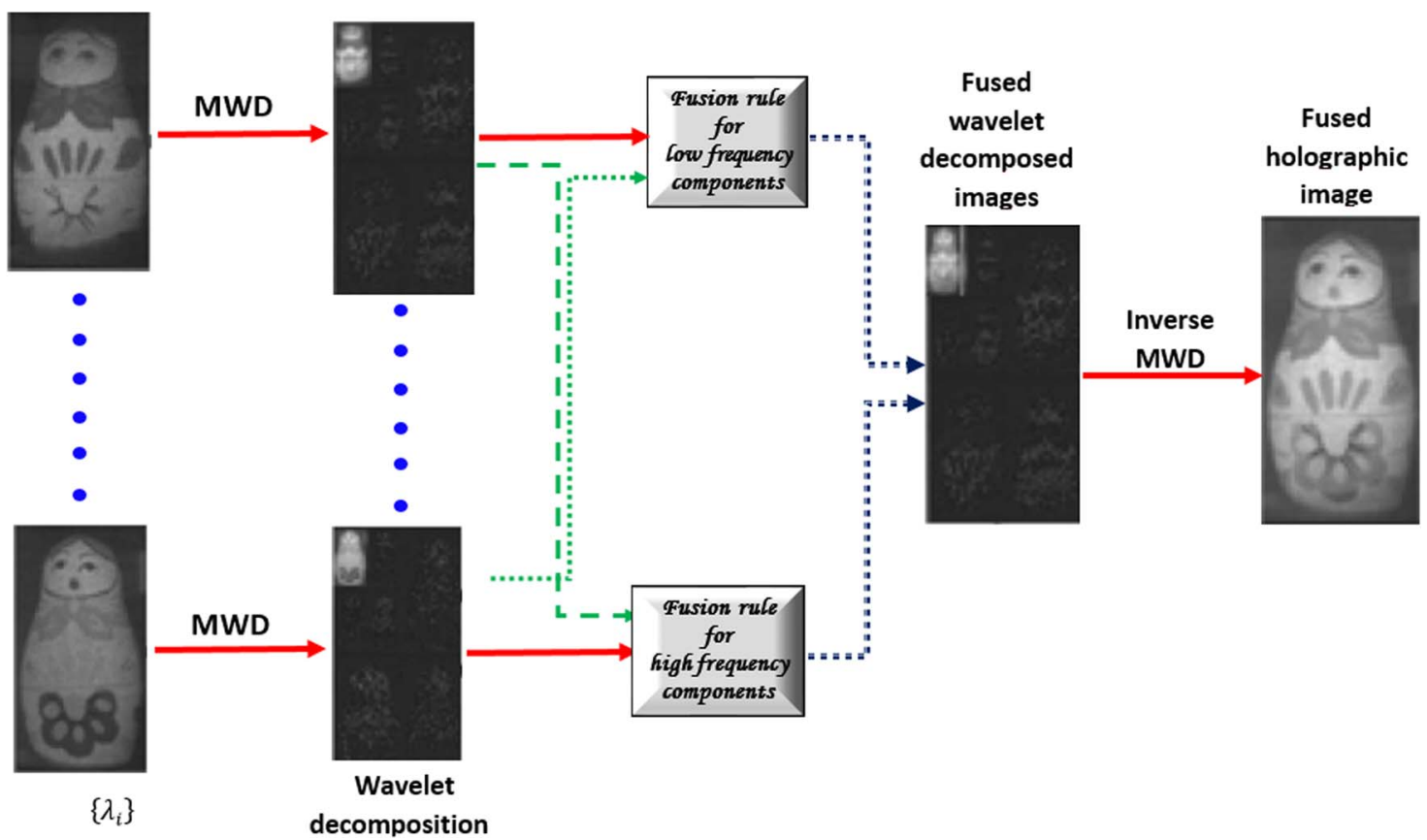

Fig. 14. Image fusion of multiwavelength holographic images by use of MWD (adapted from [102]). 


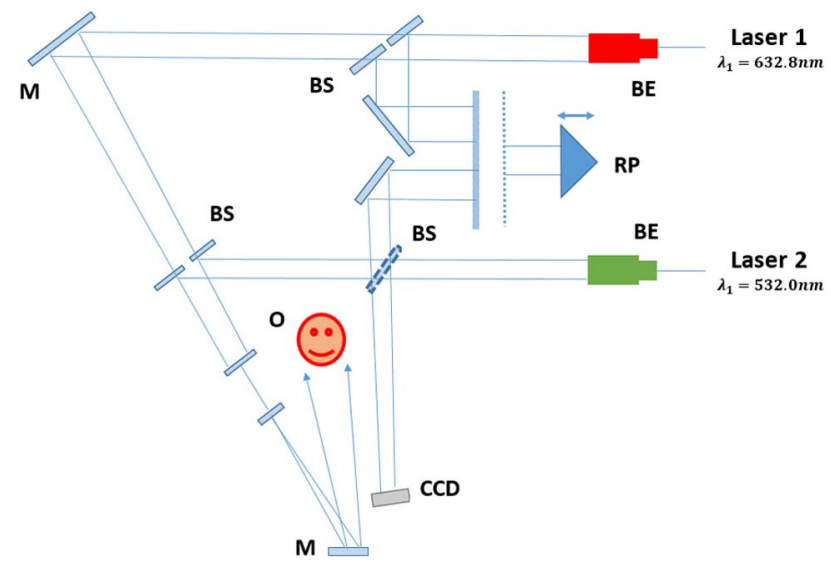

Fig. 15. Optical recording setup for digital holograms with two wavelengths. M: mirror; BE: beam expander; O: object; BS: beam splitter; RP: reflecting prism (adapted from [102]).

front of the CCD array. The CCD sensor size is $1024 \times 1024$ square pixels, $6.7-\mu \mathrm{m}$ length.

Two holograms corresponding to the two wavelengths used were reconstructed separately applying the Fresnel transformation method. Reconstruction was obtained considering the pixel size $(\Delta \xi, \Delta \eta)$ of the CCD array, being this size different from the pixel size $\left(\Delta x^{\prime}, \Delta y^{\prime}\right)$ in the image plane. They are, however, related by the following expressions: $\Delta x^{\prime}=d \cdot \lambda / N \cdot \Delta \xi$ and $\Delta y^{\prime}=d \cdot \lambda / N \cdot \Delta \eta$, where $N^{2}$ defines the total number of pixels in the CCD array and $d$ is the reconstruction distance. On the other hand, the size of the image in MWDH can be controlled and made independent of the wavelength used to record it, in order to permit a perfect superposition. In particular, $N$ was increased by padding zeros, taking into account that the following relationship is assumed to hold:

$$
N_{2} \approx N_{1}\left(\frac{\lambda_{2}}{\lambda_{1}}\right)
$$

A group of images were obtained with the two wavelengths shown in Fig. 16. They were converted into the same size using (21) and fused afterwards using a six-level decomposition scheme and Haar wavelets.

Fig. 16(a) shows an RGB image of a matrioshka doll used in the experiments. Fig. 16(b) shows the fused holographic image observed when only the red or green image is considered. Fig. 16(c) shows the reconstructed color holographic image of the matrioshka doll obtained when summing the two reconstructed images. Fig. 16(c) shows low contrast quality due to the speckle contrast. A second group of images was obtained by applying a 1-pixel vertical and horizontal shift to the previous group. These two groups were subsequently fused separately and finally combined into one image, using weighted averaging. A twofold weight was given to the high-frequency components, in order to obtain

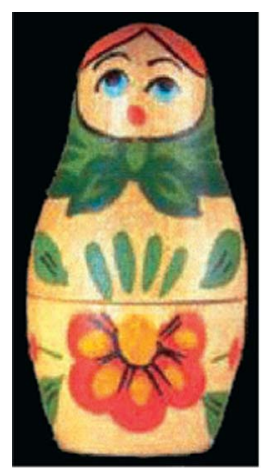

(a)

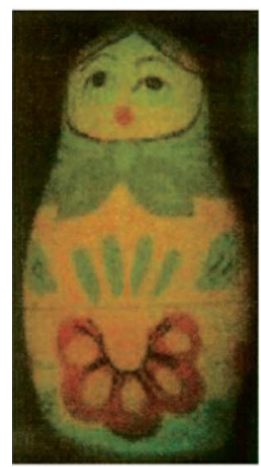

(d)

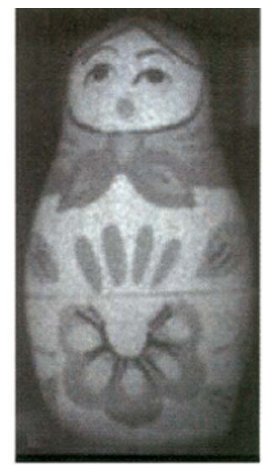

(b)

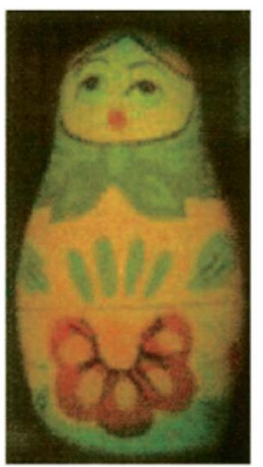

(e)

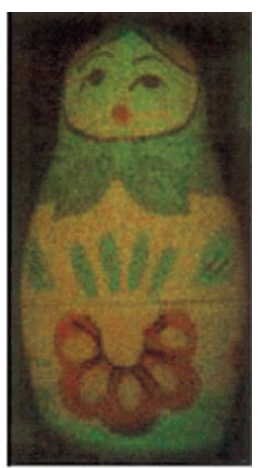

(c)

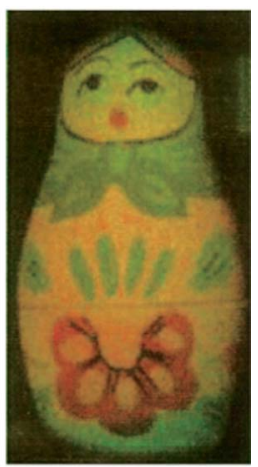

(f)
Fig. 16. Experimental holographic fusion results. (a) Original RGB image of a matrioshka doll. (b) Holographic image of red and green images fused using the MWD procedure. (c) Color holographic reconstruction obtained by summing $R G B$ composed images with $\lambda_{1}=632.8 \mathrm{~nm}$ and $\lambda_{2}=\mathbf{5 3 2 . 0} \mathbf{n m}$. (d) Fused image reconstruction. (e) Fused reconstruction after the application of the mean filter. (f) Mean-filtering result of (c) (originally from [102]).

speckle-free images. Fig. 16(d) shows the results of the weighted average fusion. The contrast improves in this case. Fig. 16(e) shows the result of the application of a $3 \times 3$ mean filter, before the fusion step. This strategy shows better results than simply summing the filtered images, taking into account that no fusion operation was applied [Fig. 16(f)].

\section{B. Multidimensional Optical Sensor and Imaging System}

Three-dimensional imaging systems allow us to obtain 3-D information about a scene. On the other hand, the use (integration) of information such as spectral and polarization features may also be useful for object recognition, image fusion, etc. In this section, we briefly summarize some of the fusion results obtained with the multidimensional optical sensor and imaging system (MOSIS) presented in [103]. Fig. 17 shows a scheme of the proposed MOSIS system. This system uses the moving array lenslet technique (MALT) to increase the reconstructed image resolution [104]. This technique allows to increase the spatial sampling rate of the acquired scene by moving the lenslet within one pitch of the lenslet. In particular, 


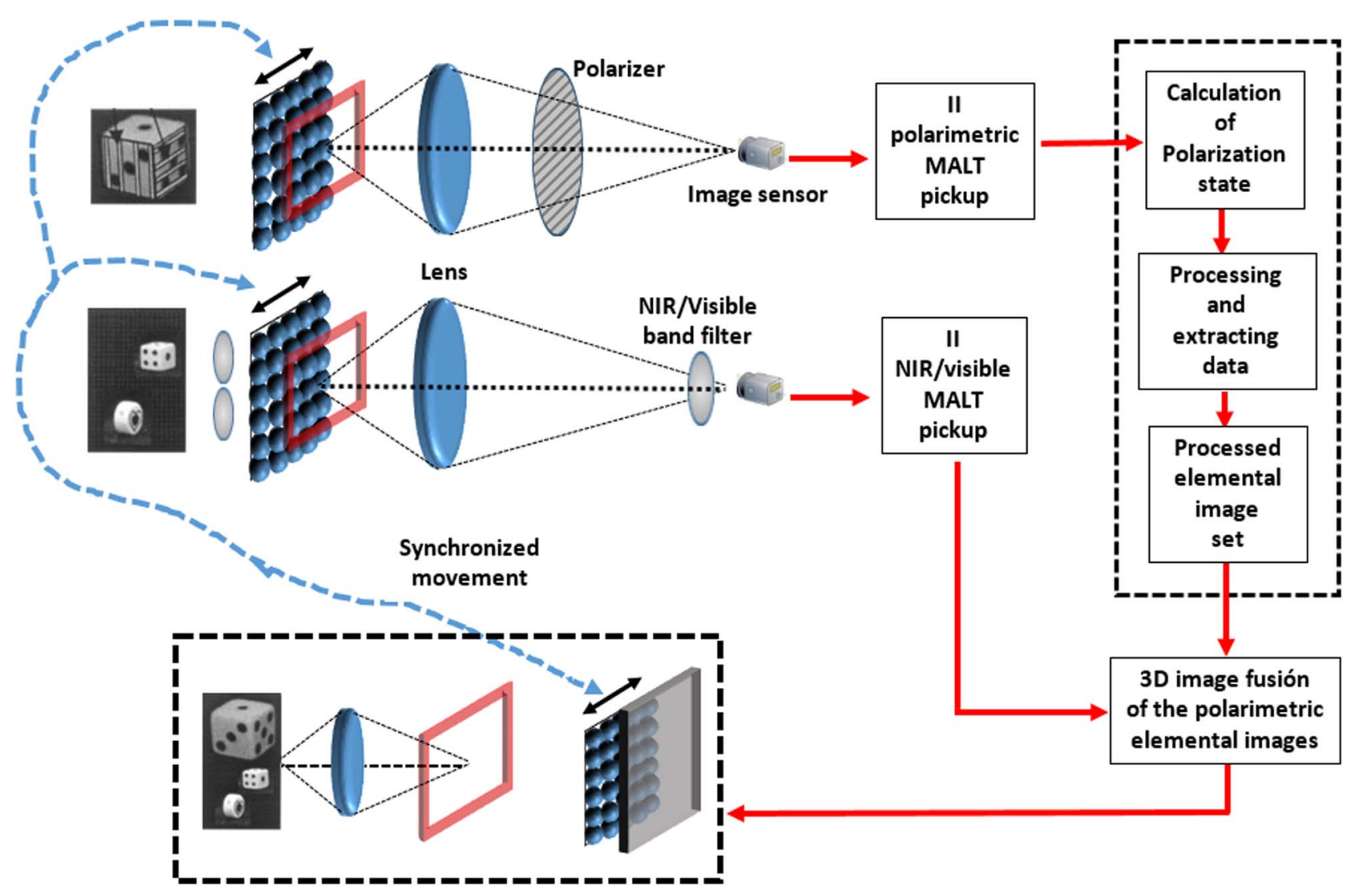

Fig. 17. Scheme of the MOSIS system with three multispectral, polarimetric, and MALT resolution capabilities (adapted from [103]).

the pickup lenslet array and the display lenslet array move synchronously with a predetermined speed in a plane that is perpendicular to the optical axis. A group of elemental image sets are recorded. These sets are integrated in the time domain afterwards, by displaying them on the spatial light modulator (SLM) or LCD at the same sampling speed of the elemental images in the pickup procedure. In computational MALT [105], the 3-D scene is reconstructed computationally with a synthesized virtual pinhole array taking into account the assessed position and intensity of the inversely mapped elemental images.

Fusion of integral images is made using wavelet decomposition. The registered images are transformed into a six-level decomposition. In the same way, as described in Section VI-A, the weighted sum fusion rule is used to fuse the high-frequency and low-frequency parts of the multiresolution wavelet coefficients. Afterwards, the corresponding fused multiresolution wavelet coefficients are inverse wavelet transformed to generate the final fused image. The MOSIS system also allows to do 3-D polarimetric imaging. A 3-D object is illuminated by linearly polarized light and the reflected light propagates through a lenslet array, and a rotating linear analyzer forms the elemental images, which are acquired afterwards by an image sensor.
The Jones vector of elliptically polarized light was subsequently obtained using a rotating linear polarizer-analyzer. On the other hand, multiwavelength information in the visible and near-infrared (NIR) range was obtained using light sources and pass-band filters (at sensor level) in the visible and NIR ranges. The visible and NIR images were finally fused/combined simultaneously to the $3-\mathrm{D}$ reconstruction.

1) Polarimetric 3-D Image Sensing and Reconstruction: Fig. 18(a)-(c) shows a die illuminated with a halogen lamp, with two orthogonally polarized sheets attached on each face. The arrows give information about the orientation of the linear analyzer-polarizer. The reflected light from the die impinged on a lenslet array. A liquid-crystal spatial light modulator (LC-SLM) in between orthogonal linear polarizers was used to change the visual characteristics of the elemental images. Additionally, the elemental images were changed to reconstruct the 3-D scene with different polarization states. In order to reconstruct the 3-D image, a polarizer and a lenslet array are placed in front of the LCD. Fig. 18(d)-(f) shows the reconstructed 3-D object with three polarization states: $\left\{0^{\circ}, 90^{\circ}, 45^{\circ}\right\}$.

2) Multidimensional Image Sensing: A scene formed by a heart-shaped object and two dice is used in the experiments. 


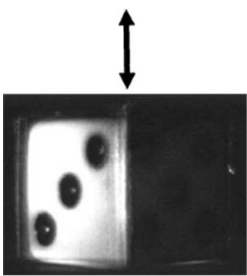

(a)

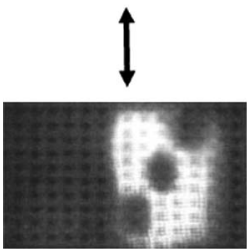

(d)

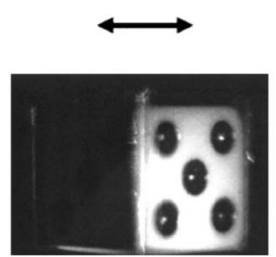

(b)

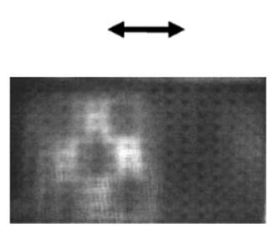

(e)

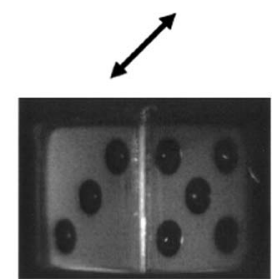

(c)

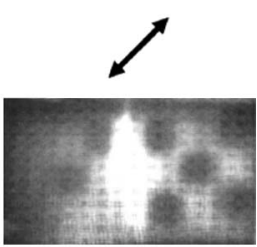

(f)
Fig. 18. Die with orthogonally polarized sheets attached to each side. Images were acquired when changing the polarization direction of the linear analyzer-polarizer: (a) vertically, (b) horizontally, and (c) diagonally. The reconstructed 3-D object for the case when the polarization direction of the linear analyzer-polarizer was (d) vertical and viewed from left, (e) horizontal and viewed from the right, and (f) diagonal and viewed from the center (adapted from [103]).

The pickup microlens array is located in front of the objects in order to form the elemental images. The distance between the front face of the die and the pickup microlens array is $19 \mathrm{~mm}$, and the distance between the heart-shaped object and the pickup microlens array is $31 \mathrm{~mm}$. A 4-megapixels $10-b$ CCD camera acquires the scene. The microlens array is composed of $53 \times 53$ square refractive microlenses (each one with a focal length of $3.3 \mathrm{~mm}$ ) in a $55-\mathrm{mm}$ square area. The size of each captured elemental image is $54 \times 54$ pixels. A $0^{\circ}$ oriented polarizing sheet is used to cover the left face of the large die and a $90^{\circ}$ oriented polarizing sheet is

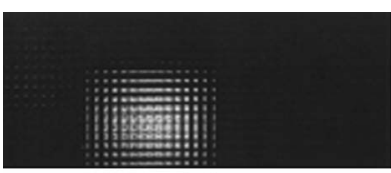

(a)

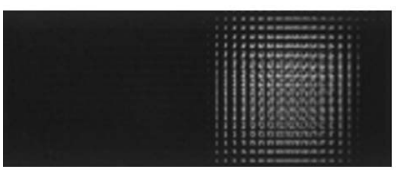

(c)

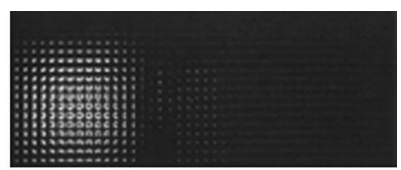

(b)

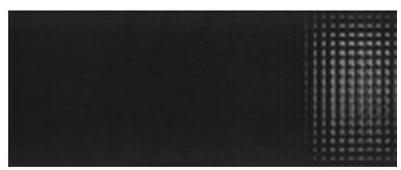

(d)
Fig. 19. Elemental images with different combinations of illumination: (a) a die illuminated by NIR light and captured by a CCD camera with an NIR pass filter; (b) a heart-shaped object illuminated by visible light and captured by a CCD camera with a visible band pass filter; (c) the left face of a die with $\mathrm{a} \mathrm{O}^{\circ}$ polarized sheet and acquired by a CCD camera with a $O^{\circ}$ polarizer; (d) the right face of a die with a $90^{\circ}$ polarized sheet and captured by a CCD camera with a $90^{\circ}$ polarizer (adapted from [103]). used to cover the right face. A visible pass and an IR pass filter with their corresponding visible and IR light sources are used to record the scene. Another polarizer was used in front of the CCD sensor. When the larger die is sensed, the small die and the heart-shaped object are covered with a polarizing sheet accordingly so that they are not detected.

3) Three-Dimensional Image Fusion of Multidimensional Data: The filters in front of the objects and the CCD camera make the objects to be sensed or not depending on the coupling between their spectrum-polarization states. Fig. 19 shows sections of the NIR-light and visible-light illuminated heart-shaped object. The scene also contains a die whose left face has a $0^{\circ}$ polarization state and the right face has a $90^{\circ}$ polarization state. The number of elemental images is $46 \times 19$ in this case. Because the heart-shaped object is illuminated by visible light, it is not seen in Fig. 19(a), and because the die is illuminated by NIR light, it is not seen in Fig. 19(b). The left and right faces can be

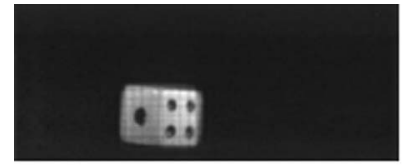

(a)

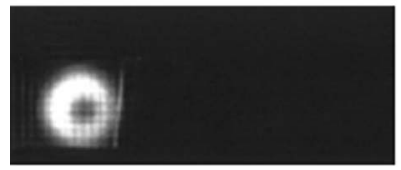

(c)

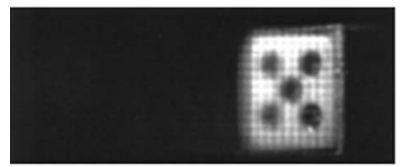

(e)

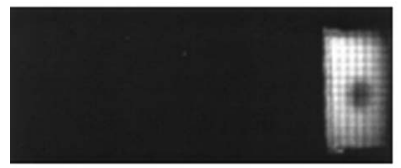

(g)

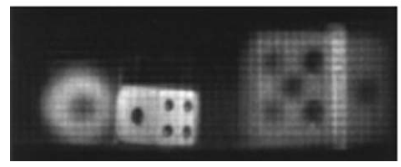

(i)

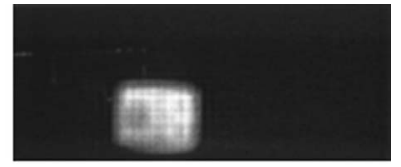

(b)

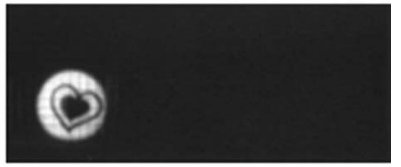

(d)

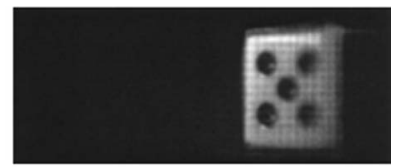

(f)

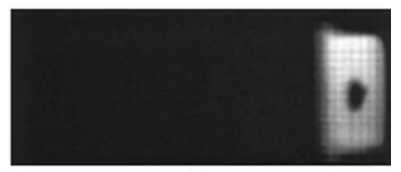

(h)

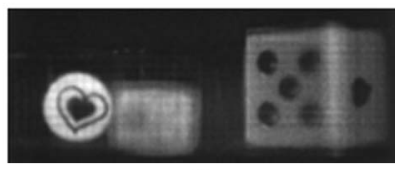

(j)
Fig. 20. Reconstructed die considering the elemental images of Fig. 19(a) at a distance of (a) z =19 mm and (b) z = $31 \mathrm{~mm}$. Reconstructed heart-shaped object image considering the elemental images that appear in Fig. 19(b) at a distance of (c) $z=19 \mathrm{~mm}$ and (d) $z=31 \mathrm{~mm}$. Reconstructed left face of a dice from the elemental images in Fig. 19(c) at a distance of (e) $z=19 \mathrm{~mm}$ and (f) $z=31 \mathrm{~mm}$. Reconstructed right face of a dice from the elemental images in Fig. 19(d) at distance of (g) $z=19 \mathrm{~mm}$ and $(\mathrm{h}) \mathrm{z}=31 \mathrm{~mm}$. Reconstructed image from the fused elemental images at a distance of (i) $z=19 \mathrm{~mm}$ and (j) z = $31 \mathrm{~mm}$ (adapted from [103]). 
seen in Fig. 19(c) and (d), respectively. The reconstructions at a depth of 19 and $31 \mathrm{~mm}$ from the elemental image in Fig. 19(a) are shown in Fig. 20(a) and (b), respectively. We can see that the die in Fig. 20(a) is in focus, whereas it is out of focus in Fig. 20(b). The reconstructions of the heartshaped object images at 19 and $31 \mathrm{~mm}$ from the elemental image in Fig. 19(b) are shown in Fig. 20(c) and (d), respectively. The reconstructed left faces (where a $0^{\circ}$ polarized sheet has been attached to it) of the larger die at 19 and $31 \mathrm{~mm}$ from the elemental image in Fig. 19(c) are shown in Fig. 20(e) and (f), respectively.

The reconstructed right faces at 19 and $31 \mathrm{~mm}$, for the larger die, when a $90^{\circ}$ polarized sheet has been attached to it [from the elemental image in Fig. 19(d)] are shown in Fig. 20(g) and (h), respectively. Images shown in Fig. 20 were normalized for visualization purposes.

Six levels of wavelet decomposition with the Haar wavelet were used, and a weighted averaging operation was applied to the decomposed high- and low-frequency components as the fusion rule. The fusion process was performed on the 2-D elemental images, and the 3-D volume was reconstructed from them. Fig. 20(i) and (j) shows these reconstructed 3-D images. With the 3-D multidimensional fusion technique, we are able to improve the image details which are not visible in the output of other sensors.

\section{CONCLUSION}

In this paper, we have presented an overview and some new results of fusion approaches applied to 3-D data obtained by 3-D imaging based on combining other sources of information, such as polarimetry and multispectral imaging, with integral imaging and digital holographic systems. In addition, the use of fusion algorithms for visualization improvement of 3-D data obtained under extremely low light illumination conditions was discussed. We have shown that fusion may result in visualization and reconstruction improvement under different acquisition conditions and visualization purposes. There is a wide variety of applications of these multiple degrees of freedom for 3-D systems ranging from healthcare, military, security, displays, entertainment, manufacturing, and night vision. The readers are referred to the references for some of these applications.

As is the case with any overview article of this nature, it has not been possible to present an exhaustive coverage of the entire fields of 3-D imaging, multimodal imaging, or fusion in this paper. Therefore, we may have inadvertently overlooked some relevant work or we did not have the scope to cover them all. We have included a number of references [35]-[105], some of which are overview articles [33], [35], [36], [38], [46], [54], to aid the readers with various aspects of these fields.

\section{REFERENCES}

[1] F. E. White, "Data fusion lexicon," presented at the Joint Directors of Laboratories, Technical Panel for C3, Data Fusion Sub-Panel, Naval Ocean Systems Center, San Diego, CA, USA, 1991.

[2] L. A. Klein, Sensor and Data Fusion Concepts and Applications, 2nd ed. Bellingham, WA, USA: SPIE, 1999.

[3] C. Debes et al., "Hyperspectral and LiDAR data fusion: Outcome of the 2013 GRSS data fusion contest," IEEE J. Sel. Top. Appl. Earth Observat. Remote Sens., vol. 7, no. 6, pp. 2405-2418, Jun. 2014.

[4] M. Dalponte, L. Bruzzone, and D. Gianelle, "Tree species classification in the Southern Alps based on the fusion of very high geometrical resolution multispectral/ hyperspectral images and LiDAR data," Remote Sens. Environ., vol. 123, pp. 258-270, 2012.

[5] H. Torabzadeh, F. Morsdorf, and M. E. Schaepman, "Fusion of imaging spectroscopy and airborne laser scanning data for characterization of forest ecosystems-A review," ISPRS J. Photogramm. Remote Sens., vol. 97, pp. 25-35, 2014.

[6] C. Pohl and J. van Genderen, "Remote sensing image fusion: An update in the context of digital Earth," Int. J. Digital Earth, vol. 7, no. 2, pp. 158-172, 2014.

[7] N. Longbotham et al., "Multi-modal change detection, application to the detection of flooded areas: Outcome of the 2009-2010 data fusion contest," IEEE J. Sel. Top. Appl. Earth Observat. Remote Sens., vol. 5, no. 1, pp. 331-342, Feb. 2012.

[8] R. Gade and T. B. Moeslund, "Thermal cameras and applications: A survey," Mach. Vis. Appl., vol. 25, pp. 245-262, 2014.
[9] V. Fernandez-Arguedas, Q. Zhang, and E. Izquierdo, "Multimodal fusion in surveillance applications," in Fusion in Computer Vision, B. Ionescu et al., Ed. New York, NY, USA: Springer-Verlag, 2014.

[10] G. Bhatnagar and Z. Liu, "A novel image fusion framework for night-vision navigation and surveillance," Signal Image Video Process., 2015, DOI:10.1007/ s11760-014-0740-6.

[11] J. Han, E. J. Pauwels, and P. de Zeeuw, "Fast saliency-aware multi-modality image fusion," Neurocomputing, vol. 111, pp. 70-80, 2013.

[12] A. P. James and B. V. Dasarathy, "Medical image fusion: A survey of the state of the art," Inf. Fusion, vol. 19, pp. 4-19, 2014.

[13] K. Uludag and A. Roebroeck, "General overview on the merits of multimodal neuroimaging data fusion," NeuroImage, vol. 102, pp. 3-10, 2014.

[14] B. H. Menze et al., "The multimodal brain tumor image segmentation benchmark (BRATS)," IEEE Trans. Med. Imaging, 2014, DOI:10.1109/TMI.2014.2377694.

[15] K. S. Tamilselvan and G. Murugesan, "Survey and analysis of various image fusion techniques for clinical CT and MRI images," Int. J. Imaging Syst. Technol., vol. 24, no. 2, pp. 193-202, 2014.

[16] P. Shah, T. V. Srikanth, S. N. Merchant, and U. B. Desai, "Multimodal image/video fusion rule using generalized pixel significance based on statistical properties of the neighborhood," Signal Image Video Process., vol. 8, no. 4, pp. 723-738, 2014.

[17] Q. Zhang, Y. Wang, M. D. Levine, X. Yuan, and L. Wang, "Multisensor video fusion based on higher order singular value decomposition," Inf. Fusion, vol. 24, pp. 54-71, 2015.
[18] H. Zhou et al., "Recent advances on singlemodal and multimodal face recognition: A survey," IEEE Trans. Human-Machine Syst., vol. 44, no. 6, pp. 701-716, Dec. 2014.

[19] N. McLaughlin, J. Ming, and D. Crookes, "Robust multimodal person identification with limited training data," IEEE Trans. Human-Machine Syst., vol. 43, no. 2, pp. 214-224, Mar. 2013.

[20] R. Raghavendra, B. Dorizzi, A. Rao, and H. Kumar, "Designing efficient fusion schemes for multimodal biometric systems using face and palmprint," Pattern Recognit., vol. 44, pp. 1076-1088, 2011.

[21] R. Raghavendran and C. Busch, "Novel image fusion scheme based on dependency measure for robust multispectral palmprint recognition," Pattern Recognit., vol. 47, pp. 2205-2221, 2014.

[22] D. Connah, M. S. Drew, and D. Finalyson, "Spectral edge image fusion: Theory and applications," in Proc. Eur. Conf. Comput. Vis., 2014, pp. 65-80.

[23] N. Brierley, T. Tippetts, and P. Cawley, "Data fusion for automated non-destructive inspection," Proc. Roy. Soc. A, vol. 470, no. 2167, 2014, DOI:10.1098/rspa.2014. 0167.

[24] B. Forster, D. Van De Ville, J. Berent, D. Sage, and M. Unser, "Complex wavelets for extended depth-of-field: A new method for the fusion of multichannel microscopy images," Microscopy Res. Tech., vol. 65, pp. 33-42, 2004.

[25] F. A. Sadjadi, "Automatic target recognition XXIII," in Proc. SPIE, vol. 8744, A. Mahalanobis, Ed., SPIE, Bellingham, WA, USA, 2013, vol. 8744 .

[26] B. Javidi, B. Javidi, and E. Tajahuerce, Multi-Dimensional Imaging, P. Andrés, Ed. New York, NY, USA: Wiley, 2014. 
[27] B. Huhle, T. Schairer, P. Jenke, and W. Straßer, "Fusion of range and color images for denoising and resolution enhancement with a non-local filter," Comput. Vis. Image Understand., vol. 114, no. 12, pp. 1336-1345, 2010.

[28] Y. Jiang and M. Wang, "Image fusion with morphological component analysis," Inf. Fusion, vol. 18, pp. 107-118, 2014.

[29] H. Yin, "Sparse representation with learned multiscale dictionary for image fusion," Neurocomputing, vol. 148, pp. 600-610, 2015.

[30] J. Sun, H. Zhu, Z. Xu, and C. Han, "Poisson image fusion based on Markov random field fusion model," Inf. Fusion, vol. 14, pp. 241-254, 2013.

[31] J. B. Sharma, K. K. Sharma, and V. Sahula, "Hybrid image fusion scheme using self-fractional Fourier functions and multivariate empirical mode decomposition," Signal Process., vol. 100, pp. 146-159, 2014.

[32] X. Bai, "Morphological image fusion using the extracted image regions and details based on multi-scale top-hat transform and toggle contrast operator," Digital Signal Process., vol. 23, no. 2, pp. 542-554, 2013.

[33] P. K. Atrey, M. A. Hossain, A. El Saddik, and M. S. Kankanhalli, "Multimodal fusion for multimedia analysis: A survey," Multimedia Syst., vol. 16, pp. 345-379, 2010.

[34] M. Wozniak, M. Graña, and E. Corchado, "A survey of multiple classifier systems as hybrid systems," Inf. Fusion, vol. 16, pp. 3-17, 2014.

[35] B. Khaleghi, A. Khamis, F. O. Karray, and S. N. Razavi, "Multisensor data fusion: A review of the state-of-the-art," Inf. Fusion, vol. 14, pp. 28-44, 2013.

[36] R. Martinez-Cuenca, G. Saavedra, M. Martinez-Corral, and B. Javidi, "Progress in 3D multiperspective display by integral imaging," Proc. IEEE, vol. 97, no. 6, pp. 1067-1077, Jun. 2009

[37] J.-Y. Son, W.-H. Son, S.-K. Kim, K.-H. Lee, and B. Javidi, "Three-dimensional imaging for creating real-world-like environments," Proc. IEEE, vol. 101, no. 1, pp. 190-205, Jan. 2013.

[38] M. Cho, M. Daneshpanah, I. Moon, and B. Javidi, "Three-dimensional optical sensing and visualization using integral imaging," Proc. IEEE, vol. 99, no. 4, pp. 556-575, Apr. 2011.

[39] T. Peterka R. L. Kooima, D. J. Sandin, A. Johnson, J. Leigh, and T. A. DeFanti, "Advances in the dynalax solid-state dynamic parallax barrier autostereoscopic visualization display system," IEEE Trans. Vis. Comput. Graphics, vol. 14, no. 3, pp. 487-499, May-Jun. 2008

[40] D. Lanman, M. Hirsch, Y. Kim, and R. Raskar, "Content-adaptive parallax barriers: Optimizing dual-layer 3D displays using low-rank light field factorization," SIGGRAPH Asia, vol. 29, no. 6, pp. 163:1-163:10, 2010.

[41] J. Arai et al., "Integral three-dimensional television using a 33-megapixel imaging system," J. Display Technol., vol. 6, no. 10, pp. 422-430, 2010

[42] F. Okano, J. Arai, K. Mitani, and M. Okui, "Real-time integral imaging based on extremely high resolution video system," Proc. IEEE, vol. 94, no. 3 , pp. 490-501, Mar. 2006.

[43] H. H. Tran et al., "Augmented reality system for oral surgery using 3D stereoscopic visualization," Medical Image Computing and Computer-Assisted Intervention-MICCAI 2011, vol. 6891. Berlin, Germany: Springer-Verlag, 2011, pp. 81-88, ser. Lecture Notes in Computer Science.

[44] H. Liao, T. Inomata, I. Sakuma, and T. Dohi, "3D augmented reality for MRI-guided surgery using integral videography autostereoscopic image overlay," IEEE Trans. Biomed. Eng., vol. 57, no. 6, pp. 1476-1486, Jun. 2010.

[45] H. Navarro, M. Martinez-Corral, G. Saavedra-Tortosa, and B. Javidi, "Is it worth using an array of cameras to capture the spatio-angular information of a 3D scene or is it enough with just two?" Proc. SPIE-Int. Soc. Opt. Eng., vol. 8384, 2012, DOI:10.1117/12.923140.

[46] A. Stern and B. Javidi, "Three-dimensional image sensing, visualization, processing using integral imaging," Proc. IEEE, vol. 94, no. 3, pp. 591-607, Mar. 2006.

[47] C. B. Burckhardt, "Optimum parameters and resolution limitation of integral photography," J. Opt. Soc. Amer., vol. 58, pp. 71-76, 1968.

[48] T. Okoshi, "Optimum design and depth resolution of lens-sheet and projection-type three-dimensional displays," Appl. Opt., vol. 10, pp. 2284-2291, 1971.

[49] L. Yang, M. McCornick, and N. Davies, "Discussion of the optics of a new 3D imaging system," Appl. Opt., vol. 27, pp. 4529-4534, 1988.

[50] S.-H. Hong, J.-S. Jang, and B. Javidi, "Three dimensional volumetric object reconstruction using computational integral imaging," Opt. Exp., vol. 3, pp. 483-491, 2004.

[51] M. Cho and B. Javidi, "Three-dimensional visualization of objects in turbid water using integral imaging," IEEE/OSA J. Display Technol., vol. 6, no. 10, pp. 544-547, Oct. 2010.

[52] Y. Zhao, X. Xiao, M. Cho, and B. Javidi, "Tracking of multiple objects in unknown background using Bayesian estimation in 3D space," J. Opt. Soc. Amer. A, vol. 28, no. 9, pp. 1935-1940, 2011.

[53] B. Javidi and F. Okano, Three Dimensional Imaging, Visualization, Display, J. Y. Son, Ed. New York, NY, USA: Springer-Verlag, 2009.

[54] X. Xiao, B. Javidi, M. Martinez-Corral, and A. Stern, "Advances in three-dimensional integral imaging: Sensing, display, applications," Appl. Opt., vol. 52, no. 4, pp. 546-560, 2013.

[55] H. Navarro, M. Martínez-Corral, G. Saavedra, A. Pons, and B. Javidi, "Photoelastic analysis of partially occluded objects with an integral-imaging polariscope," IEEE J. Display Technol., vol. 10, no. 4, pp. 255-262, Apr. 2014

[56] H. Hua and B. Javidi, "A 3D integral imaging optical see-through head-mounted display," Opt. Exp., vol. 22, no. 11, pp. 13484-13491, 2014.

[57] A. Stern, D. Aloni, and B. Javidi, "Experiments with three-dimensional integral imaging under low light levels," IEEE Photon., vol. 4, no. 4, pp. 1188-1195, Aug. 2012.

[58] D. Aloni, A. Stern, and B. Javidi, "Three-dimensional photon counting integral imaging reconstruction using penalized maximum likelihood expectation maximization," Opt. Exp., vol. 19, pp. 19 681-19 687, 2011.
[59] B. Tavakoli, B. Javidi, and E. Watson, "Three dimensional visualization by photon counting computational integral imaging," Opt. Exp., vol. 16, pp. 4426-4436, 2008.

[60] C. Lee, I. Moon, and B. Javidi, "Photon counting 3D integral imaging with compression of elemental images," J. Opt. Soc. Amer. A, vol. 29, pp. 854-860, 2012.

[61] S. Yeom, B. Javidi, and E. Watson, "Photon counting passive 3D image sensing for automatic target recognition," Opt. Exp., vol. 13, pp. 9310-9331, 2005.

[62] I. Moon and B. Javidi, "Three-dimensional recognition of photon starved events using computational integral imaging and statistical sampling," Opt. Lett., vol. 34, pp. 731-733, 2009.

[63] I. Moon, I. Muniraj, and B. Javidi, "3D visualization at low light levels using multispectral photon counting integral imaging," IEEE/OSA J. Display Technol., vol. 9, no. 1, pp. 51-55, Jan. 2013.

[64] H. Malvar, L. He, and R. Cutler, "High-quality linear interpolation for demosaicing of Bayer-patterned color images," in Proc. IEEE Int. Conf. Acoust. Speech Signal Process., 2004, vol. 3, pp. 485-488.

[65] I. Quinzán et al., "Band selection in spectral imaging for non-invasive melanoma diagnosis," Biomed. Opt. Exp., vol. 4, no. 4, pp. 514-519, 2013.

[66] S. Ahn, A. J. Chaudhuri, F. Darvas, C. A. Bouman, and R. M. Leahy, "Fast iterative image reconstruction methods for fully 3D multispectral bioluminiscence tomography," Phys. Med. Biol., vol. 53, pp. 3921-3942, 2008.

[67] J. F. Andresen, J. Busck, and H. Heiselberg, "Pulsed Raman fiber laser and multispectral imaging in three dimensions," Appl. Opt., vol. 45, no. 24, pp. 6198-6204, 2006.

[68] M. A. Powers and C. C. Davis, "Spectral LADAR: Active range-resolved three dimensional imaging spectroscopy," Appl. Opt., vol. 51, no. 10, pp. 1468-1478, 2012.

[69] A. Wallace, C. Nichol, and I. Woodhouse, "Recovery of forest canopy parameters by inversion of multispectral LiDAR data," Remote Sens., vol. 4, pp. 509-531, 2012.

[70] R. Hartley and A. Zisserman, Multiple View Geometry in Computer Vision. New York, NY, USA: Cambridge Univ. Press, 2000.

[71] X. Xiao, M. Daneshpanah, and B. Javidi, "Occlusion removal using depth mapping in three-dimensional integral imaging," IEEE/OSA J. Display Technol., vol. 8, no. 8, pp. 483-490, Aug. 2012.

[72] S. Pertuz, D. Puig, and M. A. García, "Analysis of focus measure operators for shape-from-focus," Pattern Recognit., vol. 46, pp. 1415-1432, 2013.

[73] P. Latorre Carmona et al., "Multispectral integral imaging acquisition and processing using a monochrome camera and a liquid crystal tunable filter," Opt. Exp., vol. 20, no. 23, pp. 25960-25969, 2012.

[74] X. Yu, J. Hong, C. Liu, and M. K. Kim, "Review of digital holographic microscopy for three-dimensional profiling and tracking," Opt. Eng., vol. 53, 2014, Art. ID. 112306.

[75] A. El Mallahi, C. Minetti, and F. Dubois, "Automated three-dimensional detection and classification of living organisms using digital holographic microscopy with partial spatial coherent source: Application to the monitoring of drinking water resources," Appl. Opt., vol. 52, pp. A68-A80, 2013. 
This article has been accepted for inclusion in a future issue of this journal. Content is final as presented, with the exception of pagination.

[76] Y. Frauel, T. J. Naughton, O. Matoba, E. Tajahuerce, and B. Javidi, "Three-dimensional imaging and processing using computational holographic imaging," Proc. IEEE, vol. 94, no. 3, pp. 636-653, Mar. 2006.

[77] J. Gang, "Three-dimensional display technologies," Adv. Opt. Photon., vol. 5, pp. 456-535, 2013.

[78] H. J. Coufal, D. Psaltis, G. T. Sincerbox, and A. M. Glass, Holographic Data Storage, M. J. Cardillo, Ed. New York, NY, USA: Springer-Verlag, 2000.

[79] E. Tajahuerce and B. Javidi, "Encrypting three-dimensional information with digital holography," Appl. Opt., vol. 39, no. 35, pp. 6595-6601, 2000.

[80] B. Javidi and E. Tajahuerce, "Three-dimensional object recognition by use of digital holography," Opt. Lett., vol. 25, no. 9, pp. 609-612, 2000.

[81] U. Schnars and W. P. O. Juptner, "Digital recording and numerical reconstruction of holograms," Meas. Sci. Technol., vol. 13, 2002, DOI:10.1088/0957-0233/13/9/201.

[82] D. Barada et al., "Dual-channel polarization holography: A technique for recording two complex amplitude components of a vector wave," Opt. Lett., vol. 37 pp. 4528-4530, 2012.

[83] T. Ochiai et al., "Angular multiplex recording of data pages by dual-channel polarization holography," Opt. Lett., vol. 38, pp. 748-750, 2013.

[84] T. Nomura and B. Javidi, "Object recognition by use of polarimetric phase-shifting digital holography," Opt. Lett., vol. 32, no. 15, pp. 2146-2148, 2007.

[85] T. Nomura, B. Javidi, S. Murata, E. Nitanai, and T. Numata, "Polarization imaging of a three-dimensional object by use of on-axis phase-shifting digital holography," Opt. Lett., vol. 32, no. 5, pp. 481-483, 2007.

[86] P. Ferraro et al., "Extended focused image in microscopy by digital holography," Opt. Exp., vol. 13, pp. 6738-6749, 2005.
[87] M. Paturzo et al., "Super-resolution in digital holography by a two-dimensional dynamic phase grating," Opt. Exp., vol. 16, pp. 17 107-17 118, 2008.

[88] P. Memmolo et al., "SPADEDH: A sparsity-based denoising method of digital holograms without knowing the noise statistics," Opt. Exp., vol. 20, pp. 17 250-17 257, 2012.

[89] Y. Sando, D. Barada, and T. Yatagai, "Holographic 3D display observable for multiple simultaneous viewers from all horizontal directions by using a time division method," Opt. Lett., vol. 39, pp. 5555-5557, 2014.

[90] M. K. Kim, "Full color natural light holographic camera," Opt. Exp., vol. 21, no. 8, pp. 9636-9642, 2013.

[91] P. Xia et al., "Digital holography using spectral estimation technique," IEEE/OSA $J$. Display Technol., vol. 10, no. 3, pp. 235-242, Mar. 2014.

[92] S. De Nicola et al., "Recovering correct phase information in multiwavelength digital holographic microscopy by compensation for chromatic aberrations," Opt. Lett., vol. 30, pp. 2706-2708, 2005.

[93] S. Yeom et al., "Three-dimensional color object visualization and recognition using multi-wavelength computational holography," Opt. Exp., vol. 15, pp. 9394-9402, 2007.

[94] P. Ferraro et al., "Quantitative phase microscopy of microstructures with extended measurement range and correction of chromatic aberrations by multiwavelength digital holography," Opt. Exp., vol. 15, pp. 14 591-14 600, 2007.

[95] M. P. Georges et al., "Combined holography and thermography in a single sensor through image-plane holography at thermal infrared wavelengths," Opt. Exp., vol. 22, pp. 25 517-25 529, 2014.

[96] A. K. Singh, A. Faridian, P. Gao, G. Pedrini, and W. Osten, "Quantitative phase imaging using a deep UV LED source," Opt. Lett., vol. 39, pp. 3468-3471, 2014.

[97] P. Bao, F. Zhang, G. Pedrini, and W. Osten, "Phase retrieval using multiple illumination wavelegths," Opt. Lett., vol. 33, pp. 309-311, 2008.

[98] A. Faridian et al., "Nanoscale imaging using deep ultraviolet digital holographic microscopy," Opt. Exp., vol. 18, pp. 14 159-14 164, 2010.

[99] T. Kiire, D. Barada, J.-I. Sugisaka, Y. Hayasaki, and T. Yatagai, "Color digital holography using a single monochromatic imaging sensor," Opt. Lett., vol. 37, pp. 3153-3155, 2012.

[100] B. Javidi, C. Do, S. Hong, and T. Nomura, "Multi-spectral holographic three-dimensional image fusion using discrete wavelet transform," IEEE $J$. Display Technol., vol. 2, no. 4, pp. 411-417, Dec. 2006.

[101] W. Osten et al., "Recent advances in digital holography," Appl. Opt., vol. 53, no. 27, pp. G44-G63, 2014.

[102] B. Javidi et al., "Three-dimensional image fusion by use of multiwavelength digital holography," Opt. Lett., vol. 30, no. 2, pp. 144-146, 2005.

[103] B. Javidi, S.-H. Hong, and O. Matoba, "Multidimensional optical sensor and imaging system," Appl. Opt., vol. 45, no. 13, pp. 2986-2994, 2006.

[104] J.-S. Jang and B. Javidi, "Improved viewing resolution of three-dimensional integral imaging by use of nonstationary micro-optics," Opt. Lett., vol. 27, pp. 324-326, 2002.

[105] S.-H. Jong and B. Javidi, "Improved resolution 3D object reconstruction using computational integral imaging with time multiplexing," Opt. Exp., vol. 12, pp. 4579-4588, 2004.

\section{ABOUT THE AUTHORS}

Pedro Latorre-Carmona received the B.S. degree in physics from the University of Valencia, Valencia, Spain, in 1999 and the Ph.D. degree in computer science from the Polytechnical University of Valencia, Valencia, Spain, in 2005.

$\mathrm{He}$ is a Postdoctoral Researcher at the Departamento de Lenguajes y Sistemas Informaticos, Universitad Jaume I, Castellón de la Plana, Spain. His current research interests are feature selection and extraction, pattern recognition, 3-D and multispectral (including remote sensing) image processing, colorimetry, and vision physics.

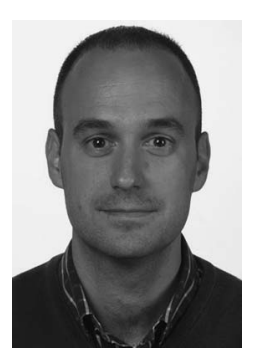

Filiberto Pla received the B.Sc. degree in physicselectronics and the Ph.D. degree in computer science.

He is a Full Professor at the Departament de Llenguatges i Sistemes Informàtics, University Jaume I, Castellón de la Plana, Spain. He received the BSC in Physics-Electronics and the PhD in Computer Science. He has been a Visiting Scientist at Silsoe Research Institute, the University of Surrey, and the University of Bristol in the United

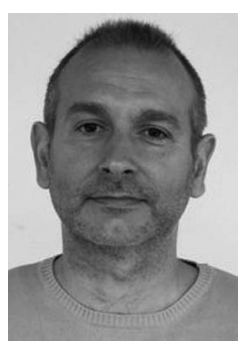
Kingdom; CEMAGREF in France; the University of Genoa in Italy; Instituto Superior Técnico of Lisbon in Portugal; and the Swiss Federal Institute of Technology ETH-Zurich and the idiap Research Institute in Switzerland. Currently, he is the Director of the Institute of New Imaging Technologies, University Jaume I. His current research interests are color and spectral image analysis, visual motion analysis, 3-D image visualization, and pattern recognition techniques applied to image processing.

Dr. Pla is a member of the Spanish Association for Pattern Recognition and Image Analysis (AERFAl), which is a part of the International Association for Pattern Recognition (IAPR). 
Adrian Stern (Member, IEEE) received the B.Sc., M.Sc. (cum laude), and Ph.D. degrees in electrical and computer engineering from Ben-Gurion University of the Negev, Beer Sheva, Israel, in 1988, 1997, and 2002 respectively.

$\mathrm{He}$ is an Associate Professor at the ElectroOptical Engineering Department, Ben-Gurion University of the Negev. During 2002-2004, he was a Postdoctoral Fellow at the University of Connecticut, Storrs, CT, USA. During 2007-2008, he served

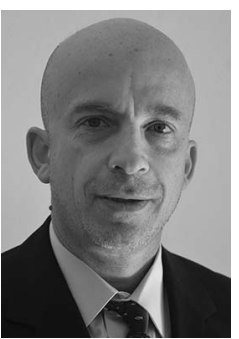
as a Senior Research and Algorithm Specialist for GE Molecular Imaging, Israel. Currently, during his sabbatical leave, he is holding a visiting teaching and scholar position at the Massachusetts Institute of Technology, Cambridge, MA, USA. His current research interests include 3-D imaging, compressive imaging, and computational imaging.

Dr. Stern is a Fellow of the International Society for Optics and Photonics (SPIE) and a member of the Optical Society of America (OSA). He serves as an Associate Editor for Optics Express, and has served as Guest Editor for the IEEE/OSA JouRnAL ON DISPLAY TECHNOLOGY.

Inkyu Moon (Member, IEEE) received the B.S. and M.S. degrees in electronics engineering from SungKyunKwan University, Seoul, Korea, in 1996 and 1998, respectively, and the M.S. and Ph.D. degrees in electrical and computer engineering from the University of Connecticut, Storrs, CT. USA, in 2007.

He joined Chosun University, Gwangju, Korea, in 2009, and is currently an Associate Professor at the Department of Computer Engineering. He has

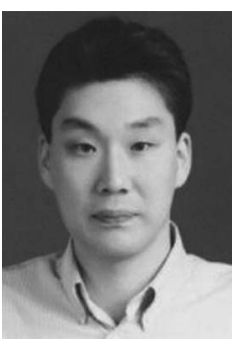
over 100 publications, including over 50 peer-reviewed journal articles, over 50 conference proceedings, including over ten keynote addresses, and invited conference papers. His research interests include digital holography, biomedical imaging, and optical information processing.

Dr. Moon is a member of the Optical Society of America (OSA) and the International Society for Optics and Photonics (SPIE). He is on the Editorial Board of the Korea Multimedia Society.
Bahram Javidi (Fellow, IEEE) received the B.S. degree in electrical engineering from George Washington University, Washington, DC, USA and the M.S. and Ph.D. degrees in electrical engineering from Pennsylvania State University, University Park, PA, USA.

$\mathrm{He}$ is the Board of Trustees Distinguished Professor at the University of Connecticut, Storrs, CT, USA. He has over 900 publications, including over 400 peer-reviewed journal articles, over

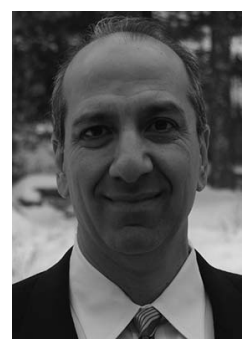
400 conference proceedings, including over 110 plenary addresses, keynote addresses, and invited conference papers. His interests are in a broad range of transformative imaging approaches using optics and photonics, and he has made contributions to passive and active multidimensional imaging from nanoscale to microscale and macroscale. His recent research activities include 3-D visualization and recognition of objects in photon-starved environments using passive imaging; automated disease identification using biophotonics with low-cost compact sensors for use in developing countries; information security, encryption, and authentication using quantum imaging; nonplanar flexible 3-D image sensing, and bioinspired imaging.

Dr. Javidi is a Fellow of the Optical Society of America (OSA) and the International Society for Optics and Photonics (SPIE). In 2015, he was the recipient of the European Physical Society Prize for Applied Aspects of Quantum Electronics and Optics. In 2010, he received The George Washington University's Distinguished Alumni Scholar Award, University's highest honor for its alumni in all disciplines. In 2008, he received the Fellow award by John Simon Guggenheim Foundation. He was the recipient of the 2008 IEEE Donald G. Fink prized paper award among all (over 130) IEEE Transactions, Journals, and Magazines. In 2007, The Alexander von Humboldt Foundation awarded Dr. Javidi with Humboldt Prize for outstanding U.S. scientists. He received the Technology Achievement Award from SPIE in 2008. In 2005, he received the Dennis Gabor Award in Diffractive Wave Technologies from SPIE. He was the recipient of the IEEE Photonics Distinguished Lecturer Award twice in 2003-2004 and 2004-2005. He was awarded the IEEE Best Journal Paper Award from the IEEE TRANSACTIONS ON VeHICULAR TECHNOLOGY twice in 2002 and 2005. Early in his career, the National Science Foundation named Prof. Javidi a Presidential Young Investigator, and he received The Engineering Foundation and the IEEE Faculty Initiation Award. He was selected in 2003 as one of the nation's top 160 engineers between the ages of 30-45 by the National Academy of Engineering (NAE) to be an invited speaker at The Frontiers of Engineering Conference which was cosponsored by The Alexander von Humboldt Foundation. He became an alumnus of the Frontiers of Engineering of The National Academy of Engineering in 2003. He has served on the Editorial Board of the Proceedings of THE IEEE [ranked \#1 among all electrical engineering journals], the advisory board of the IEEE PHOTONICS JOURNAL, and he was on the founding Board of Editors of the IEEE/OSA JOURNAL OF DISPLAY TECHNOLOGY. His papers have been cited over $28000+$ according to Google Scholar (h index = 78; i10 index $=407$ ). He is the coauthor of nine papers that received best paper awards. 